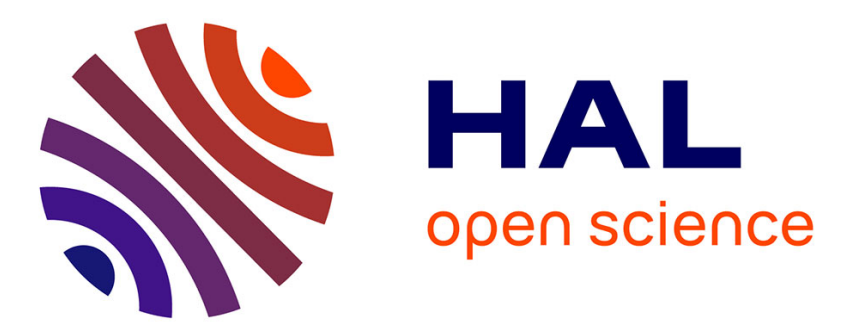

\title{
Optimal synthesis for the minimum time control problems of fed-batch bioprocesses for growth functions with two maxima
}

Térence Bayen, Pedro Gajardo, Francis Mairet

\section{- To cite this version:}

Térence Bayen, Pedro Gajardo, Francis Mairet. Optimal synthesis for the minimum time control problems of fed-batch bioprocesses for growth functions with two maxima. Journal of Optimization Theory and Applications, 2013, 158 (2), pp.521-553. 10.1007/s10957-012-0225-0 . hal-00655534v3

HAL Id: hal-00655534

https://hal.science/hal-00655534v3

Submitted on 2 Apr 2012

HAL is a multi-disciplinary open access archive for the deposit and dissemination of scientific research documents, whether they are published or not. The documents may come from teaching and research institutions in France or abroad, or from public or private research centers.
L'archive ouverte pluridisciplinaire HAL, est destinée au dépôt et à la diffusion de documents scientifiques de niveau recherche, publiés ou non, émanant des établissements d'enseignement et de recherche français ou étrangers, des laboratoires publics ou privés. 


\title{
Optimal synthesis for the minimum time control problems of fed-batch bioprocesses for growth functions with two maxima
}

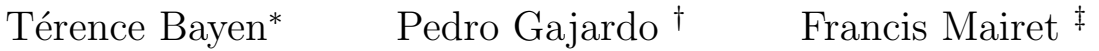

April 2, 2012

\begin{abstract}
We address the problem of finding an optimal feedback control for feeding a fed-batch bioreactor with one species and one substrate from a given initial condition to a given target value in a minimal amount of time. Recently, the optimal synthesis (optimal feeding strategy) has been obtained in systems in which the microorganisms involved are represented by increasing growth functions or growth functions with one maxima, with either Monod or Haldane functions, respectively (widely used in bioprocesses modeling). In the present work, we allow impulsive controls corresponding to instantaneous dilutions, and we assume that the growth function of the microorganism present in the process has exactly two local maxima. This problem has been considered from a numerical point of view in [15] without impulsive controls. In this article, we introduce two singular arc feeding strategies, and we define explicit regions of initial conditions in which the optimal strategy is either the first singular arc strategy or the second strategy.
\end{abstract}

Keywords. optimal control, minimal time problem, impulsive control, Pontryagin maximum principle, bioreactor.

MSC. 49J15, 49K15, 49N25.

\section{Introduction}

The operation of a fed-batch bioreactor consists of feeding the reactor with a highly concentrated solution without any removal of liquid before the end of the culture. This operating mode presents many advantages over batch or chemostat (continuous culture) operations: a high concentration of cells or products can be obtained, and it allows for the easy control of the operating conditions, in particular the limiting substrate concentration (see [9]). Therefore, fed-batch bioreactors have been widely used in the food and pharmaceutical industries, as well as in wastewater treatment. In a fed-batch process, choosing an adequate feeding strategy is a key feature that can significantly increase productivity. Many authors have proposed feeding strategies using optimal control theory for various types of problems (different bioprocesses and/or objectives, see for example [8] and [10]). In this study, we will focus on the minimal time problem: given initial conditions, the objective is to define a feeding strategy to reach a given substrate concentration in a minimal amount of time with a given maximal volume (representing a completely full tank). The optimal synthesis for this problem has been obtained in [13] using Green's theorem, via the technique introduced in [11], in the following cases:

- For increasing growth functions (e.g., the Monod kinetic, see [12, 17]), the optimal solution is of the Bang-Bang type: it consists of filling the reactor up to the maximum volume with the maximum input flow rate and then waiting until the substrate concentration reaches the desired value.

- For nonmonotonic growth functions with one maximum point (e.g., Haldane kinetic, see [1, 17]), the optimal solution is a singular arc strategy, consisting of regulating the substrate concentration at the maximum of the specific growth rate.

\footnotetext{
*Université Montpellier 2, Case courrier 051, 34095 Montpellier cedex 5, France (tbayen@math.univ-montp2.fr)

†Departamento de Matemática, Universidad Técnica Federico Santa María, Avenida España 1680, Valparaíso, Chile (pedro.gajardo@usm.cl)

¥Departamento de Matemática, Universidad Técnica Federico Santa María, Avenida España 1680, Valparaíso, Chile (francis.mairet@usm.cl)
} 
These results, obtained for regular controls in [13], have been recently extended to allow for impulsive controls (corresponding to instantaneous dilutions), in [7], for the following situations :

- for the case of one microbial species with increasing (e.g., Monod) or one maxima (e.g., Haldane) growth functions,

- for the case in which two microbial species compete for the same substrate with two increasing growth functions that satisfy additional assumptions.

Several new methods have also been proposed for the practical implementation of these optimal strategies (see [3]), including alternative approaches such as extremum seeking (see [5]). In this work, we will focus on the problem of fed-batch operation with growth functions having two local maxima. This type of growth function is observed when the bacterial population can grow via two possible metabolic pathways (e.g., each one is related to a different non-limiting substrate). Indeed, for some microbial species, a high substrate concentration can result in the changing of metabolic pathways (see [6]). A growth function having two local maxima can also be obtained when we consider a growth function with one maximum point associated with inhibition by a product. Under some hypotheses, the product concentration can be expressed as a function of the substrate concentration, and the problem can be rewritten with a growth function with two local maxima (see $[2]$ ).

In [15], the problem of minimal time control for a growth function with two local maxima has been addressed using a numerical approach. It appears that the singular arcs defined by the two local maxima are two optimal candidates, but to obtain the optimal synthesis is not easy, because none of the previous methods applied for Monod or Haldane growth function are applicable here. Given the numerical results obtained using a regularization method, the authors conjecture that the state space can be divided into two domains in which each arc strategy is optimal. To solve this problem analytically, we allow impulsive control (see [7]). In general, an impulsive control indicates that the input flow rate is allowed to take values $u_{m} \gg 1$ corresponding to instantaneous dilution. We consider two cases, which depend on whether the strict global maximum of the growth function is the first local maximum (case B) or the second maximum (case A). The problem is reformulated into a planar affine system with two controls (as was done in [13] without impulsive control) introducing a constant $M$ defined by the initial conditions (see Section 2). We then use the Pontryagin maximum principle (see [14]) to derive the necessary conditions on optimal trajectories. Our method relies on the exclusion of extremal trajectories that have a switching point before reaching one of the two singular arcs. We then conclude by considering which singular strategy starting from a given initial condition has the minimal cost. The paper is organized as follows. In Section 2, the problem is stated, and we derive several general properties about extremal trajectories via the Pontryagin maximum principle. In particular, we provide an explicite expression of the switching functions corresponding to the controls (see lemmas 2.3 and 2.4), which is the starting point for comparing both strategies. These expressions allow, in particular, the easy retrieval of a result in [7] on the optimality of the singular arc strategy in the case of one species with a Haldane growth function. The original proof (see [7]) makes use of the characterization of the value function by using the Hamilton-Jacobi equation (a sufficient condition). In Section 3, we prove the general comparison results between extremal trajectories that are valid for cases A and B. Section 4 and 5 are devoted to cases A and $\mathrm{B}$. The following results are obtained:

- Case $\mathrm{A}, M \geq 0$ : the optimal synthesis is given by theorem 4.1

- Case A, $M<0$ : the optimal synthesis is obtained under the hypothesis 4.1 (see theorem 4.2).

- Case B, $M \geq 0$ : we prove that the first singular arc is optimal (see theorem 5.1).

- Case B, $M<0$ : we prove that the first singular arc is optimal (theorem 5.2) provided that the hypotheses 5.1 and 5.2 are satisfied.

For $M \geq 0$, our optimal synthesis proves the conjecture proposed in [15] when impulsive control is allowed. It appears that the case $M<0$ (which corresponds to a low initial concentration of biomass) is slightly more delicate to handle than the case $M \geq 0$. In case $\mathrm{B}, M<0$, hypothesis 5.1 is satisfied when $M$ is small. Hypotheses 4.1 and 5.2 have been verified numerically (see Figure 4). 


\section{Statement of the problem and general results}

\subsection{Formulation of the problem}

In this subsection, we present the model and the optimal control problem. Let us consider the following control system describing a perfectly mixed reactor operated in fed-batch mode with impulsive control (see [7]):

$$
\left\{\begin{array}{l}
\dot{x}=\left(r \mu(s)-\frac{u}{v}\right) x, \\
\dot{s}=-r \mu(s) x+\frac{u}{v}\left(s_{i n}-s\right), \\
\dot{v}=u
\end{array}\right.
$$

where $x$ is the concentration of biomass, $s$ is the concentration of substrate, and $v$ is the volume of water (with concentrations $x$ and $s$ ) in the tank. We will assume that the growth function $\mu$ has two local maxima $s_{1}<s_{2}$ and one local minimum $s_{m} \in\left(s_{1}, s_{2}\right)$, see Figure 1 . We will consider two cases:

- Case A: $\mu\left(s_{1}\right)<\mu\left(s_{2}\right)$.

- Case B: $\mu\left(s_{1}\right)>\mu\left(s_{2}\right)$.

We also suppose that $\mu^{\prime \prime}\left(s_{1}\right) \neq 0, \mu^{\prime \prime}\left(s_{2}\right) \neq 0$, and $\mu^{\prime \prime}\left(s_{m}\right) \neq 0$, and we define $s_{2}^{\prime} \in\left[s_{m}, s_{2}\right]$ such that $\mu\left(s_{2}^{\prime}\right)=\mu\left(s_{1}\right)$ in case A, and similarly, $s_{1}^{\prime} \in\left[s_{1}, s_{m}\right]$ such that $\mu\left(s_{1}^{\prime}\right)=\mu\left(s_{2}\right)$ in case B.

The set of admissible controls is defined as follows:

$$
\mathcal{U}=\{\mathbf{u}=(r, u):[0, \infty[\rightarrow(\{0,1\} \times[0,1]) \backslash\{(0,0)\} \mid \text { meas. }\} .
$$

Here, $u$ is the input flow rate and $r$ represents an impulse control. An instantaneous addition of volume $v_{+}-v_{-}$(i.e., a jump from volume $v_{-}$to volume $v_{+}$) is achieved by taking $r=0$ on some interval of time $\left[\tau_{-}, \tau_{+}\right]$for system $(2.1)$ and any measurable control $u$ satisfying

$$
\int_{\tau_{-}}^{\tau_{+}} u(t) d t=v_{+}-v_{-},
$$

see [7] for more details. In particular, there is no uniqueness of $u$ as long as integral (2.2) is equal to $v_{+}-v_{-}$. An addition of volume $v_{+}-v_{-}$corresponds to a dilution of the substrate and the biomass:

$$
s_{+}=\frac{v_{-}}{v_{+}} s_{-}+\left(1-\frac{v_{-}}{v_{+}}\right) s_{i n}, x_{+}=\frac{v_{-}}{v_{+}} x_{-},
$$

where $s_{-}, x_{-}$are the concentrations before dilution and $s_{+}, x_{+}$are the concentrations after dilution. We
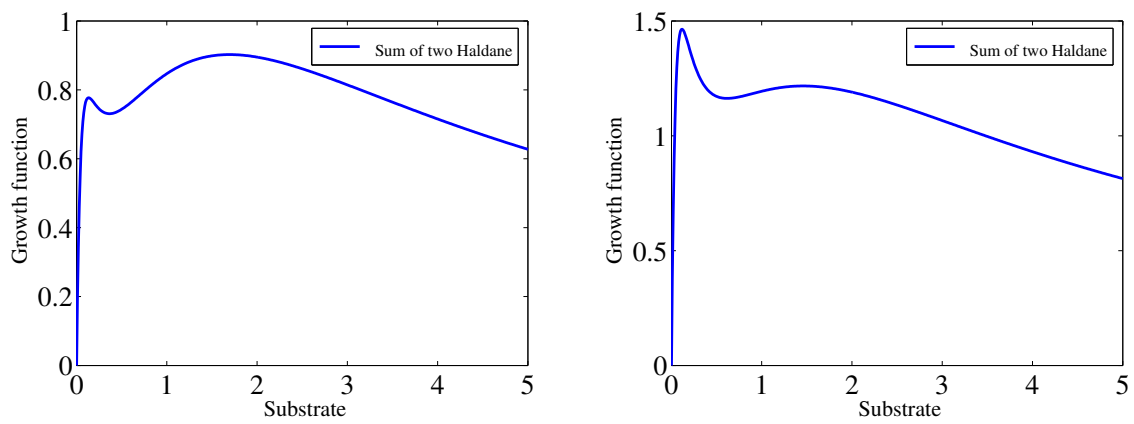

Figure 1: Figure left: example of a growth function (corresponding to case A) that is a sum of two Haldane growth functions having two local maxima $s_{1}<s_{2}$, such that $\mu\left(s_{1}\right)<\mu\left(s_{2}\right)$. Figure right: sum of two Haldane growth functions with $\mu\left(s_{1}\right)>\mu\left(s_{2}\right)$ (case B).

denote by $\xi$ a triple $\xi=(x, s, v)$, and let $\left.\left.E=\mathbb{R}_{+}^{*} \times\right] 0, s_{i n}[\times] 0, v_{m}\right] \subset \mathbb{R}^{3}$ be the domain of state variables $x, s$, and $v$. The target is defined as follows:

$$
\mathcal{T}=\left\{\xi=(x, s, v) \in E \mid 0 \leq s \leq s_{\text {ref }}, v=v_{m}\right\},
$$


where $s_{\text {ref }}$ is a given reference (low) concentration such that $0<s_{\text {ref }}<s_{1}<s_{2}<s_{\text {in }}$, and $v_{m}$ is the volume of the tank. For $\xi \in E$ and a control $\mathbf{u} \in \mathcal{U}$, let $t_{\xi}(\mathbf{u})$ be the first entry time in $\mathcal{T}$. In the impulsive framework, the minimum time problem, for an initial condition $\xi_{0} \in E$, can be gathered into:

$$
\inf _{\mathbf{u} \in \mathcal{U}} \int_{0}^{t_{\xi_{0}}(\mathbf{u})} r(\tau) d \tau, \text { s.t. }(x(0), s(0), v(0))=\xi_{0} ;
$$

see [7] for more details on the reparametrization of the minimum time problem with impulsive control. If $(x, s, v)$ is a solution of $(2.1)$, the function $M:=v\left(x+s-s_{i n}\right)$ is constant equal to $v_{0}\left(x_{0}+s_{0}-s_{\text {in }}\right)$; hence, we have $x=\frac{M}{v}-\left(s-s_{i n}\right)$, and the system can be gathered into a quasi-affine planar system with two inputs:

$$
\left\{\begin{array}{l}
\dot{s}=-r \mu(s)\left(\frac{M}{v}-\left(s-s_{i n}\right)\right)+\frac{u}{v}\left(s_{i n}-s\right), \\
\dot{v}=u
\end{array}\right.
$$

Remark 2.1. The sign of the constant $M=v_{0}\left(x_{0}+s_{0}-s_{i n}\right)$ defined by the initial conditions plays an important role in the synthesis of the optimal feeding strategy. Note that for repeated fed-batch cultures (i.e., at the end of the culture, the reactor is partially drained, and then a new culture begins using the substrate and biomass concentrations reached at the end of the previous culture), one can easily check that $M$ will tend to 0.

We claim that for any $\xi_{0} \in E$, the minimum time to reach $\mathcal{T}$ is finite. Indeed, one can reach volume $v_{m}$ from any $\xi_{0} \in E$ by an impulse of volume $v_{m}-v_{0}$ (that is, $r=0$ ). If the concentration of substrate is greater than $s_{\text {ref }}$, we put $u=0$ and $r=1$ until $s(t) \leq s_{\text {ref }}$. This strategy steers any $\xi_{0} \in E$ to the target $\mathcal{T}$ in finite time, which proves that (2.3) is finite. The existence of an optimal solution of (2.3) is not guaranteed by Filipov's theorem (see [4]), as the set of admissible controls is not convex (recall that $r(\cdot) \in\{0,1\}$ ). Let us now consider the relaxed problem, that is, the problem $(2.3)$ in which the control $(r, u)$ is allowed to take values within $U:=[0,1] \times[0,1]$. By the convexity of $U$ and the cost functional, the existence of an optimal control for the relaxed problem is standard by Filipov's theorem, see [4]. Without loss of generality, we may consider trajectories such that $(r, u) \neq 0$ (if $r=u=0$, the cost is unchanged while the system is stationary). We will see in the next section that an optimal control of the relaxed problem can be taken such that $r(\cdot) \in\{0,1\}$.

\subsection{Pontryagin maximum principle}

In this subsection, we derive the necessary conditions for an optimal control by the Pontryagin maximum principle (PMP), and we compute the singular arcs. We also prove the existence of an optimal solution of problem (2.3). Let $\gamma_{0}$ be the curve, in the plane $s-v$, defined by:

$$
\gamma_{0}(s)=v_{m}\left(\frac{s_{i n}-s_{r e f}}{s_{i n}-s}\right), s \in\left[0, s_{r e f}\right] .
$$

In the rest of the paper, we will consider initial conditions for the system (2.4) in the set $\mathcal{D}$ defined as follows:

$$
\mathcal{D}=\left\{\left(s_{0}, v_{0}\right) \in \mathbb{R}_{+}^{*} \times \mathbb{R}_{+}^{*} \mid s_{0} \in\left(0, s_{i n}\right), 0<v_{0}<\min \left(\gamma_{0}\left(s_{0}\right), v_{m}\right), M+v_{0}\left(s_{\text {in }}-s\right)>0\right\} .
$$

Note that the last condition guarantees that the initial conditions $\xi_{0}$ of system (2.1) satisfy $x_{0}>0$. We will prove in the next subsection that for initial conditions $\left(s_{0}, v_{0}\right)$ such that $\gamma_{0}\left(s_{0}\right) \leq v_{0} \leq v_{m}$, the optimal control is a single impulse from volume $v_{0}$ to volume $v_{m}$ (see proposition 2.3). Therefore, we will assume throughout the paper that initial conditions are in $\mathcal{D}$. The Hamiltonian of the system $H=H\left(s, v, \lambda_{s}, \lambda_{v}, \lambda_{0}, r, u\right)$ is:

$$
H=-r \lambda_{s} \mu(s)\left(\frac{M}{v}-\left(s-s_{i n}\right)\right)+u\left(\frac{\lambda_{s}\left(s_{i n}-s\right)}{v}+\lambda_{v}\right)+\lambda_{0} r .
$$

Let us apply the Pontryagin maximum principle to the relaxed problem. Let $(r, u)$ be an optimal solution and $(s, v)$ the corresponding solution of (2.4). Then, there exists $t_{f}>0, \lambda_{0} \leq 0$ and an absolutely continuous map $\lambda=\left(\lambda_{s}, \lambda_{v}\right):\left[0, t_{f}\right] \rightarrow \mathbb{R}^{2}$ such that $\left(\lambda_{0}, \lambda\right) \neq 0, \dot{\lambda}_{s}=-\frac{\partial H}{\partial s}, \dot{\lambda}_{v}=-\frac{\partial H}{\partial v}$, that is:

$$
\left\{\begin{array}{l}
\dot{\lambda}_{s}=\lambda_{s}\left(r \mu^{\prime}(s) x-r \mu(s)+\frac{u}{v}\right), \\
\dot{\lambda}_{v}=\frac{\lambda_{s}}{v^{2}}\left(-r \mu(s) M+u\left(s_{i n}-s\right)\right),
\end{array}\right.
$$


and we have the maximality condition:

$$
\text { for a.e. } t \in\left[0, t_{f}\right], H\left(s(t), v(t), \lambda_{s}(t), \lambda_{v}(t), \lambda_{0}, r(t), u(t)\right)=\max _{(\tilde{r}, \tilde{u}) \in U} H\left(s(t), v(t), \lambda_{s}(t), \lambda_{v}(t), \lambda_{0}, \tilde{r}, \tilde{u}\right)=0 \text {. }
$$

The Hamiltonian in (2.9) is zero, as $t_{f}$ is free. Without loss of generality, we may assume $\lambda_{0}=-1$. Next, define the switching functions $\phi_{1}$ and $\phi_{2}$ associated with controls $r$ and $u$ :

$$
\left\{\begin{array}{l}
\phi_{1}(t)=-\lambda_{s} \mu(s)\left(\frac{M}{v}-\left(s-s_{i n}\right)\right)-1 \\
\phi_{2}(t)=\lambda_{s} \frac{s_{i n}-s}{v}+\lambda_{v}
\end{array}\right.
$$

We call an extremal trajectory a quintuplet $\left(s(\cdot), v(\cdot), \lambda_{s}(\cdot), \lambda_{v}(\cdot), r(\cdot), u(\cdot)\right)$ satisfying $(2.4)-(2.8)-(2.9)$. It follows that any extremal trajectory satisfies for a.e. $t \in\left[0, t_{f}\right]$ :

$$
r(t) \phi_{1}(t)+u(t) \phi_{2}(t)=0
$$

We obtain from (2.9) that any optimal control satisfies the following control law: for a.e. $t \in\left[0, t_{f}\right]$, we have

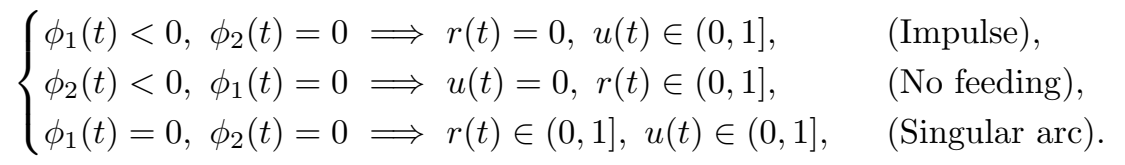

In particular, an extremal trajectory has a singular arc on some time interval $I$ if both switching functions satisfy $\phi_{1}(t)=\phi_{2}(t)=0$ for all $t \in I$. Derivating the switching functions yields:

$$
\dot{\phi}_{1}(t)=-u \phi(t), \dot{\phi}_{2}(t)=r \phi(t)
$$

where:

$$
\phi(t)=\frac{\lambda_{s} \mu^{\prime}(s)\left(s_{i n}-s\right) x}{v} .
$$

The next lemma will be useful in the following.

Lemma 2.1. If $\left(s_{0}, v_{0}\right)$ is in $\mathcal{D}$, then any optimal trajectory satisfies $\lambda_{s}<0$.

Proof. An optimal trajectory is a concatenation of arcs where $u=0$ (no feeding), $r=0$ (impulse), or $s$ is constant with $s \in\left\{s_{1}, s_{2}, s_{m}\right\}$ (singular arc, see lemma $2.2(\mathrm{i})$ ). If the initial condition is in $\mathcal{D}$, any optimal trajectory contains an arc $u=0$ (otherwise, the trajectory would not reach $\mathcal{T}$ ). If $\lambda_{s}$ vanishes at some point $t_{0} \in\left[0, t_{f}\right]$, then $\lambda_{s} \equiv 0$ from (2.8). Hence, when $u=0$, we obtain from (2.7) that $r=0$, which is not possible (recall that we assumed $(r, u) \neq(0,0))$. Hence, $\lambda_{s}$ is always non-zero and of constant sign. Moreover, when $u=0$, one has from $(2.7)-r \lambda_{s} \mu(s) x-r=0$; thus, $\lambda_{s}<0$.

From the expression of $\phi$ and the sign of $\lambda_{s}$, one can prove the following proposition that will be useful to perform the optimal synthesis in the two next sections.

Proposition 2.1. (i). Let $\left(s_{0}, v_{0}\right) \in \mathcal{D}$ such that $s_{0} \in\left(s_{m}, s_{2}\right]$ and let us consider an extremal trajectory satisfying $u=0$ at time 0 . Then, there exists $t_{1}>0$ with $s\left(t_{1}\right)<s_{m}$ such that we have $u=0$ on $\left[0, t_{1}\right]$.

(ii). Let $\left(s_{0}, v_{0}\right) \in \mathcal{D}$ such that $s_{0} \in\left[s_{1}, s_{m}\right), v_{0}<v_{m}$, and let us consider an extremal trajectory satisfying $r=0$ at time 0 . Then, there exists $t_{1}^{\prime}>0$ with $s\left(t_{1}^{\prime}\right)>s_{m}$ such that we have $r=0$ on $\left[0, t_{1}^{\prime}\right]$.

Proof. Let us prove (i). From (2.14), we have that $\phi$ is non-negative when $s(t) \in\left[s_{1}, s_{m}\right]$, and $\phi$ is negative when $s(t) \in\left[s_{m}, s_{2}\right]$. It follows that if $s(t) \in\left[s_{1}, s_{m}\right]$, then $\phi_{2}$ is increasing, whereas if $s(t) \in\left[s_{m}, s_{2}\right]$, then $\phi_{2}$ is decreasing. When $u=0$, the concentration of substrate $s(t)$ is strictly decreasing at time 0 ; thus, $\phi_{2}$ remains negative until $s(t)=s_{m}$. If $\phi_{u}$ is always negative, then the trajectory does not reach the target (as $\left.v_{0}<v_{m}\right)$. Therefore, there exists $t_{1}>0$ with $s\left(t_{1}\right)<s_{m}$ such that $\phi_{2}\left(t_{1}\right)=0$. The proof of (ii) is similar using $\phi_{1}(0) \leq 0$ and the monotonicity of $\phi_{1}$ for $s(t) \in\left[s_{1}, s_{m}\right]$.

The previous lemma means that an arc $r=0$ (resp. an arc $u=0$ ) starting with $s_{0} \in\left[s_{1}, s_{m}\right.$ ) (resp. $\left.s_{0} \in\left(s_{m}, s_{2}\right]\right)$ always passes though the line $s=s_{m}$. Let us now evaluate a singular arc strategy. 
Lemma 2.2. Let us consider a trajectory that contains a singular arc on some time interval $\left[t_{0}, t_{1}\right]$. Then:

(i). The concentration of substrate is constant on $\left[t_{0}, t_{1}\right]$, and $s \in\left\{s_{1}, s_{2}, s_{m}\right\}$.

(ii). If the singular arc is optimal, then $s \in\left\{s_{1}, s_{2}\right\}$.

(iii). The cost of a singular arc from a volume $v_{0}$ to a volume $v_{1}$ is:

$$
\int_{t_{0}}^{t_{1}} r(t) d t=\frac{1}{\mu\left(s_{i}\right)} \ln \left(\frac{M+v_{1}\left(s_{i n}-s_{i}\right)}{M+v_{0}\left(s_{i n}-s_{i}\right)}\right), i=1,2,
$$

and the singular control can be expressed as follows:

$$
u_{s_{i}}=\mu\left(s_{i}\right)\left(v+\frac{M}{s_{i n}-s_{i}}\right), i=1,2
$$

Proof. By the definition of a singular arc, we have $\phi_{1}(t)=\phi_{2}(t)=0$ on $\left[t_{0}, t_{1}\right]$. By derivating, we obtain $\phi(t)=0$ for $t \in\left[t_{0}, t_{1}\right]$ as $(r, u) \neq(0,0)$. In view of $(2.14)$, the only possibility for $\phi$ to be zero on $\left[t_{0}, t_{1}\right]$ is $\mu^{\prime}(s)=0$; hence, $s$ must be constant on the interval $\left[t_{0}, t_{1}\right]$, that is, $s=s_{1}, s=s_{m}$ or $s=s_{2}$, which proves (i). By using $\dot{s}=0$ on $\left[t_{0}, t_{1}\right],(2.4)$ becomes:

$$
\dot{v}-r \mu(s) v=r \mu(s) \frac{M}{s_{i n}-s} .
$$

Solving this equation yields:

$$
v_{1}=\left(v_{0}+\frac{M}{s_{i n}-s}\right) e^{\mu(s) \int_{t_{0}}^{t} r(\tau) d \tau}-\frac{M}{s_{i n}-s},
$$

and we obtain (2.15). By derivating, we obtain (2.16), which proves (iii). From (2.15), we may assume that $r=1$ along a singular arc. Indeed, it is enough to ensure that the $L^{1}$-norm of $r$ on some time interval (where the volume goes from $v_{0}$ to $v_{1}$ ) is equal to the right hand side of $(2.15)$.

Let us now discuss the optimality of a singular arc. By derivating $\phi(t)$ and replacing $r(t)$ by 1 , we obtain:

$$
\phi^{\prime}(t)=\frac{\lambda_{s} x}{v}\left(\mu^{\prime \prime}(s)\left(s_{i n}-s\right)\left(-\mu(s) x+\frac{u}{v}\left(s_{i n}-s\right)\right)+x \mu(s) \mu^{\prime}(s)-2 \frac{u}{v} \mu^{\prime}(s)\left(s_{i n}-s\right)+\mu^{\prime}(s) x\right) .
$$

An optimal control $u$ must satisfy the Legendre-Clebsch necessary condition along the trajectory, that is, $\frac{\partial}{\partial u} \frac{d^{2}}{d t^{2}} H_{u} \geq 0$, see e.g., [16]. Combining $\mu^{\prime}(s)=0$ and (2.17) yields:

$$
\frac{\partial}{\partial u} \frac{d^{2}}{d t^{2}} H_{u}=\mu^{\prime \prime}(s) \frac{\lambda_{s} x\left(s_{i n}-s\right)^{2}}{v} .
$$

Thus, one must have $\mu^{\prime \prime}(s) \leq 0$ (recall that $\lambda_{s}<0$ ). Hence, the only possibility for $s$ is $s=s_{1}$ or $s=s_{2}$, which proves (ii).

Theorem 2.1. There exists an optimal solution of (2.3) such that $r \in\{0,1\}$.

Proof. Let $(r, u) \neq(0,0)$ an optimal solution of the relaxed problem. Notice that $(r, u)$ satisfies $(2.12)$ for a.e. $t \in\left[0, t_{f}\right]$. In the case of an impulse, we have $r=0$, whereas in the case of a singular arc, one can take $r=1$ by the previous lemma. Let us now examine the case where $u=0$ (no feeding). If $u=0$ on some time interval $\left[t_{0}, t_{1}\right]$, the volume $v$ is constant, and we have $\dot{s}=r(t) f(s(t))$, where $f(s)=-\mu(s)\left(M / v-\left(s-s_{\text {in }}\right)\right)$. Let us put

$$
\left\{\begin{array}{l}
\theta=\int_{t_{0}}^{t} r(\tau) d \tau \\
z(\theta(t))=s(t)
\end{array}\right.
$$

By derivating, one has $\frac{d z}{d \theta}(\theta(t)) r(t)=\dot{s}(t)=r(t) f(s(t))$; thus, we have:

$$
\frac{d z}{d \theta}=f(z(\theta))
$$

as $r$ is non-zero. Moreover, $z$ satisfies $z(0)=s\left(t_{0}\right)$ and $z(\bar{\theta})=s\left(t_{1}\right)$, where $\bar{\theta}=\int_{t_{0}}^{t_{1}} r(\tau) d \tau$, and the cost remains unchanged (as the cost of $z$ on the interval $[0, \bar{\theta}]$ is $\bar{\theta}$ ). We can repeat this argument on any arc $u=0$ of the trajectory, which allows us to take $r=1$ by (2.18). This concludes the proof. 
Remark 2.2. Theorem 2.1 answers a question raised in [7] on the existence of an optimal solution of (2.3) with $r \in\{0,1\}$ in the case of one species. The existence of an optimal control of (2.3) with a control $r$ such that $r \in\{0,1\}$ remains an open question in presence of more than one species.

Definition 2.1. The singular arc strategy $S A S_{i}, i=1,2$ (corresponding to $s=s_{1}$ or $s=s_{2}$ ) starting at some point $\left(s_{0}, v_{0}\right) \in \mathcal{D}$ is defined as follows:

$$
\left(r\left(s_{0}, v_{0}\right), u\left(s_{0}, v_{0}\right)\right)=\left\{\begin{array}{l}
\left(1, u_{s_{i}}\right) \text { if } s_{0}=s_{i} \text { and } v_{0}<v_{m} \\
(1,0) \text { if } s>s_{i} \text { or } v_{0}=v_{m} \\
(0, \tilde{u}) \text { if } s<s_{i} \text { and } v_{0}<v_{m}
\end{array}\right.
$$

where control $\tilde{u}$ is any measurable function taking values in $[0,1]$ such that (2.2) holds in presence of a dilution.

The next proposition is concerned with some restrictions on the parameters of the system in order to obtain admissible singular arcs.

Proposition 2.2. (i). If $M<0$ and $\mu\left(s_{i}\right) v_{m} \leq 1$ for $i=1,2$ then both singular strategies are well-defined. (ii). If $M \geq 0$ and $\mu\left(s_{i}\right)\left(v_{m}+\frac{M}{s_{i n}-s_{i}}\right) \leq 1, i=1,2$, then both singular strategies are well-defined.

Proof. By lemma 2.2, with $i=1,2$, the control $u_{s_{i}}$ is admissible if and only if:

$$
0 \leq \mu\left(s_{i}\right)\left(v(t)+\frac{M}{s_{\text {in }}-s_{i}}\right) \leq 1, t \in\left[t_{0}, t_{1}\right] .
$$

As $\left\{\left(s_{0}, v_{0}\right) \in \mathbb{R}_{+}^{*} \times \mathbb{R}_{+}^{*} \mid s_{0} \in\left(0, s_{i n}\right), 0<v_{0} \leq v_{m}, M+v_{0}\left(s_{i n}-s\right)>0\right\}$ is invariant by the dynamics, and the left inequality in (2.19) is always satisfied. When $M<0$, a sufficient condition to have an admissible control is $\mu\left(s_{i}\right) v_{m} \leq 1, i=1,2$ (to ensure the right inequality of (2.19)). This proves (i). The proof of (ii) is similar.

Remark 2.3. From a practical point of view, we assume that the parameters of the system always satisfy the conditions given by the previous proposition.

\subsection{Computations of the switching functions}

In this subsection, we compute the switching functions in the case of non-feeding (that is $u=0$ and $r=1$ ) and in the case of an impulse $(r=0)$ in order to address the global optimality of the singular arc strategies $S A S_{i}, i=1,2$ in the next section. Let us define a function $\psi_{v}$ by:

$$
\psi_{v}(s)=\frac{s_{i n}-s}{M+v\left(s_{i n}-s\right)}
$$

Notice that $\psi_{v}$ is non-negative by (2.4). In the case where $M=0$, one has $\psi_{v}(s)=\frac{1}{v}$. Moreover, one has $\psi_{v}^{\prime}(s)=\frac{-M}{\left(M+v\left(s_{i n}-s\right)\right)^{2}}$; hence, if $M<0$, then $\psi_{v}$ is increasing, whereas if $M>0$, then $\psi_{v}$ is decreasing.

Lemma 2.3. Let us consider an extremal trajectory starting at $\left(s_{0}, v_{0}\right) \in \mathcal{D}$ at time 0 with $u=0$ on some time interval $\left[0, t_{1}\right]$. Then, we have for $t \in\left[0, t_{1}\right]$ :

$$
\begin{gathered}
\lambda_{s}(t)=\frac{-v_{0}}{\mu(s(t))\left(M+v_{0}\left(s_{i n}-s(t)\right)\right)}, \lambda_{v}(t)=\lambda_{v}(0)+\int_{s_{0}}^{s(t)} \frac{\psi_{v_{0}}^{\prime}(s)}{\mu(s)} d s, \\
\phi_{2}(t)=\lambda_{v}(0)-\frac{\psi_{v_{0}}\left(s_{0}\right)}{\mu\left(s_{0}\right)}+\int_{s_{0}}^{s(t)} \psi_{v_{0}}(s) \frac{\mu^{\prime}(s)}{\mu^{2}(s)} d s .
\end{gathered}
$$

The cost associated to this trajectory is:

$$
J=-\int_{s_{0}}^{s\left(t_{1}\right)} \frac{d s}{\mu(s)\left(\frac{M}{v_{0}}+s_{i n}-s\right)}
$$


Proof. The expression of $\lambda_{s}$ is straightforward from the expression of the Hamiltonian along $u=0$. We then obtain $\dot{\lambda}_{v}=\frac{\frac{M}{v_{0}}}{M+v_{0}\left(s_{i n}-s\right)}$. By dividing by $\dot{s}$ (which is non-zero as $(r, u) \neq(0,0)$ ), we obtain:

$$
\frac{d \lambda_{v}}{d s}=-\frac{M}{\mu(s)\left(M+v_{0}\left(s_{i n}-s\right)\right)^{2}}=\frac{\psi_{v_{0}}^{\prime}(s)}{\mu(s)},
$$

and we obtain the desired expression of $\lambda_{v}$ by integrating. Replacing $\lambda_{s}$ into the expression of $\phi$ yields:

$$
\phi_{2}(t)=\lambda_{v}(t)-\frac{\psi_{v_{0}}(s(t))}{\mu(s(t))},
$$

and we obtain the desired expression for $\phi_{2}$ by integrating by parts the expression of $\lambda_{v}$. Finally, the cost $J$ is obtained by integrating the differential equation $\dot{s}$ along $u=0$.

Let $\mathcal{D}^{\prime}=\left\{\left(s_{0}, v_{0}\right) \in \mathbb{R}_{+}^{*} \times \mathbb{R}_{+}^{*} \mid \gamma_{0}\left(s_{0}\right) \leq v_{0} \leq v_{m}\right\}$. From the previous lemma, we obtain the optimal solution for any initial conditions in $\mathcal{D}^{\prime}$.

Proposition 2.3. (i). If $\left(s_{0}, v_{0}\right) \in \mathcal{D}^{\prime}$, then the optimal feedback steering $\left(s_{0}, v_{0}\right)$ to the target is an impulse of volume $v_{m}-v_{0}$.

(ii). An extremal trajectory starting at some point $\left(s_{0}, v_{0}\right) \in \mathcal{D}$ such that $s_{0} \leq s_{1}, v_{0}<v_{m}$ and satisfying $u=0$ on a time interval $\left[0, t_{1}\right]$ is not optimal.

Proof. (i). If $v_{0}=v_{m}$, then the initial point is in $\mathcal{T}$, and the result is proved. Assume $v_{0}<v_{m}$. If an optimal trajectory satisfies $u=0$ with volume $v<v_{m}$ on some time interval $\left[t_{0}, t_{1}\right]$, we must have $\phi_{2}\left(t_{0}\right) \leq 0$, and there exists a time $t_{0}^{\prime}>0$ such that $\phi_{2}\left(t_{0}^{\prime}\right)=0$ (otherwise, the trajectory would not reach $\mathcal{T}$ ). We obtain:

$$
\phi_{2}\left(t_{0}\right)<\phi_{2}\left(t_{0}^{\prime}\right)=0=\phi_{2}\left(t_{0}\right)+\int_{s\left(t_{0}\right)}^{s\left(t_{0}^{\prime}\right)} \psi_{v}(s) \frac{\mu^{\prime}(s)}{\mu^{2}(s)} d s
$$

hence, we obtain:

$$
\int_{s\left(t_{0}\right)}^{s\left(t_{0}^{\prime}\right)} \psi_{v}(s) \frac{\mu^{\prime}(s)}{\mu^{2}(s)} d s>0 .
$$

But one has $\psi_{v}(s)>0, \mu^{\prime}(s)>0$ (recall that $s<s_{1}$ ), and $s\left(t_{0}\right)>s\left(t_{0}^{\prime}\right)$. Consequently, the integral above is negative, which is a contradiction. We can use the same argument for proving (ii).

Corollary 2.1. Consider an optimal trajectory starting from a point $\left(s_{0}, v_{0}\right) \in \mathcal{D}$ such that $0<s_{0} \leq s_{2}$ and $0<v_{0}<v_{m}$. Assume that $u=0$ in the neighborhood of zero.

(i). There exists a time $t_{0}$ such that $\phi_{2}\left(t_{0}\right)=0, s\left(t_{0}\right)<s_{m}$, and we have:

$$
\int_{s\left(t_{0}\right)}^{s_{0}} \psi_{v_{0}}(s) \frac{\mu^{\prime}(s)}{\mu^{2}(s)} d s<0
$$

(ii). Moreover, if $M \leq 0$, we have:

$$
\mu\left(s\left(t_{0}\right)\right)>\mu\left(s_{0}\right)
$$

Proof. We have $\phi_{2}(0)<0$; hence, $\phi_{2}$ remains negative for $t$ close to zero. As $v_{0}<v_{m}$, the switching function must be zero at a certain time, and we obtain the existence of a time $t_{0}$ such that $\phi_{2}\left(t_{0}\right)=0$. Notice that $s\left(t_{0}\right)<s_{m}$. Indeed, if $s_{0}<s_{m}$, the result is clear, as $s$ is decreasing along $u=0$. If $s_{0}>s_{m}$, the result follows from proposition 2.1. The previous conditions on the switching function can be written:

$$
\phi_{2}(0)<0=\phi_{2}\left(t_{0}\right)=\phi_{2}(0)+\int_{s\left(t_{0}\right)}^{s_{0}}-\psi_{v_{0}}(s) \frac{\mu^{\prime}(s)}{\mu^{2}(s)} d s=0,
$$

hence:

$$
\int_{s\left(t_{0}\right)}^{s_{0}}-\psi_{v_{0}}(s) \frac{\mu^{\prime}(s)}{\mu^{2}(s)} d s>0
$$


which proves (i). From this last inequality, we obtain:

$$
\int_{s_{m}}^{s_{0}} \psi_{v_{0}}(s) \frac{\mu^{\prime}(s)}{\mu^{2}(s)} d s<\int_{s\left(t_{0}\right)}^{s_{m}} \psi_{v_{0}}(s) \frac{-\mu^{\prime}(s)}{\mu^{2}(s)} d s .
$$

When $M \leq 0, \psi_{v_{0}}$ is increasing; therefore:

$$
\psi_{v_{0}}\left(s_{m}\right) \int_{s_{m}}^{s_{0}} \frac{\mu^{\prime}(s)}{\mu^{2}(s)} d s \leq \int_{s_{m}}^{s_{0}} \psi_{v_{0}}(s) \frac{\mu^{\prime}(s)}{\mu^{2}(s)} d s<\int_{s\left(t_{0}\right)}^{s_{m}} \psi_{v_{0}}(s) \frac{-\mu^{\prime}(s)}{\mu^{2}(s)} d s \leq \psi_{v_{0}}\left(s_{m}\right) \int_{s\left(t_{0}\right)}^{s_{m}} \frac{-\mu^{\prime}(s)}{\mu^{2}(s)} d s .
$$

We obtain the inequality:

$$
\psi_{v_{0}}\left(s_{m}\right)\left(\frac{1}{\mu\left(s_{m}\right)}-\frac{1}{\mu\left(s_{0}\right)}\right)<\psi_{v_{0}}\left(s_{m}\right)\left(\frac{1}{\mu\left(s_{m}\right)}-\frac{1}{\mu\left(s\left(t_{0}\right)\right)}\right),
$$

which gives (2.24).

We now compute the switching functions in the case of an impulse.

Lemma 2.4. Assume that an extremal trajectory starting at $\left(s_{0}, v_{0}\right) \in \mathcal{D}$ with $0<v_{0}<v_{m}$ satisfies $r=0$ on $\left[0, t_{0}\right]$. Then, one has:

$$
\begin{gathered}
\lambda_{s}(t)=\frac{\lambda_{s}(0)}{v_{0}} v(t), \lambda_{v}(t)=\lambda_{v}(0)+\frac{\lambda_{s}(0)}{v_{0}}\left(s-s_{0}\right), \\
\phi_{1}(t)=-\lambda_{s}(0) x_{0} \mu(s)-1, \phi_{2}(t)=\lambda_{v}(0)+\frac{\lambda_{s}(0)\left(s_{i n}-s_{0}\right)}{v_{0}}=0 .
\end{gathered}
$$

Proof. The proof is straightforward from the state-adjoint equation and the Hamiltonian. Notice that the adjoint vector must satisfy $\lambda_{v}(0)+\frac{\lambda_{s}(0)\left(s_{i n}-s_{0}\right)}{v_{0}}=0$ to ensure $\phi_{2}=0$ along the impulse.

Remark 2.4. The cost associated to this trajectory is null.

Lemma 2.5. Assume that an extremal trajectory starting at $\left(s_{0}, v_{0}\right) \in \mathcal{D}$ with $0<v_{0}<v_{m}$ satisfies $r=0$ on $\left[0, t_{0}\right]$ and $\phi_{1}\left(t_{0}\right)=0$ for $t_{0}>0$. Then, one has:

$$
\mu\left(s\left(t_{0}\right)\right) \geq \mu\left(s_{0}\right)
$$

Proof. Condition $\phi_{1}(0) \leq 0$ gives $\mu\left(s_{0}\right) \leq \frac{1}{-\lambda_{s}(0) x_{0}}$, and condition $\phi_{1}\left(t_{0}\right)=0$ gives $-\lambda_{s}(0) x_{0} \mu\left(s\left(t_{0}\right)\right)-1=0$; hence, $\mu\left(s\left(t_{0}\right)\right)=\frac{1}{-\lambda_{s}(0) x_{0}} \geq \mu\left(s_{0}\right)$.

The previous lemma has simple consequences:

- In case A, any trajectory starting at $s_{0} \geq s_{2}$ with an impulse is not optimal, as $\mu$ is strictly decreasing on $\left[s_{2},+\infty\right)$. This remark (combined with proposition 2.3 (ii)) allows us to retrieve the optimality of a singular strategy for one species and a Haldane growth function in the impulsive case, see [7] (the proof in [7] makes use of the value function and the Hamilton-Jacobi equation).

- In case A, any extremal trajectory starting at $\left(s_{0}, v_{0}\right) \in \mathcal{D}, s_{0} \in\left[s_{1}, s_{m}\right]$ with an impulse, is such that $\phi_{1}$ will vanish between $s_{0}^{\prime}$ and $\min \left(s_{2}, s^{*}\left(s_{0}, v_{0}\right)\right)$ where $s_{0}^{\prime} \in\left[s_{m}, s_{2}\right]$ satisfies $\mu\left(s_{0}^{\prime}\right)=\mu\left(s_{0}\right)$ and $s^{*}\left(s_{0}, v_{0}\right)$ is defined by $s^{*}\left(s_{0}, v_{0}\right)=s_{0} \frac{v_{0}}{v_{m}}+\left(1-\frac{v_{0}}{v_{m}}\right) s_{i n}$. Indeed, combining lemma 2.5 and the monotonicity of $\mu$ on $\left[s_{2},+\infty\right)$, we have that an extremal trajectory crossing $s=s_{2}$ with an impulse is not optimal.

- In case B, any trajectory starting at $s_{1} \leq s_{0}<s_{1}^{\prime}$ with an impulse is not optimal by using (2.28). 


\section{General comparison results for case $\mathrm{A}$ and $\mathrm{B}$}

In this section, we compare the cost of several extremal trajectories in order to exclude some candidates for optimality. Let us define:

$$
s_{1}^{*}=\left\{\begin{array}{ll}
s_{1} & \text { for case A, } \\
s_{1}^{\prime} & \text { for case } \mathrm{B},
\end{array} \quad \text { and } s_{2}^{*}= \begin{cases}s_{2}^{\prime} & \text { for case A, } \\
s_{2} & \text { for case } \mathrm{B}\end{cases}\right.
$$

and let $\gamma_{2}^{*}(s), \gamma_{2}(s)$, such that:

$$
\gamma_{2}^{*}(s)=v_{m}\left(\frac{s_{i n}-s_{2}^{*}}{s_{i n}-s}\right), \gamma_{2}(s)=v_{m}\left(\frac{s_{i n}-s_{2}}{s_{i n}-s}\right) .
$$

Notice that for $s \in] 0, s_{2}[$ :

$$
\gamma_{2}(s)<\gamma_{2}^{*}(s) \text { for case A, and } \gamma_{2}(s)=\gamma_{2}^{*}(s) \text { for case B. }
$$

Let us now consider $\mathcal{C}_{2}$ and $\mathcal{C}_{2}^{*}$ defined by:

$$
\mathcal{C}_{2}=\left\{(s, v) \in\left(0, s_{2}\right] \times\left(0, v_{m}\right] \mid v=\gamma_{2}(s)\right\}, \mathcal{C}_{2}^{*}=\left\{(s, v) \in\left(0, s_{2}\right] \times\left(0, v_{m}\right] \mid v=\gamma_{2}^{*}(s)\right\} .
$$

The curves $\mathcal{C}_{2}$ and $\mathcal{C}_{2}^{*}$ are dilution curves; hence, if $\left(s_{0}, v_{0}\right) \in \mathcal{C}_{2}$, then an impulse of volume $v-v_{0}$ (with $\left.v<v_{m}\right)$ transforms $\left(s_{0}, v_{0}\right)$ into another point of $\mathcal{C}_{2}$. It is important to see that any set

$$
F_{\bar{s}}:=\left\{(s, v) \in\left(0, s_{i n}\right) \times\left(0, v_{m}\right) \mid v \geq v_{m}\left(\frac{s_{i n}-\bar{s}}{s_{i n}-s}\right)\right\},
$$

where $\bar{s} \in\left(0, s_{i n}\right)$, is such that any extremal trajectory starting in $F_{\bar{s}}$ will stay in this set for all $t$. In the following, we make use of the notations:

- $I_{s_{-}, v_{+}}^{s_{+}, v_{+}}$denotes a dilution (or impulse) from $\left(s_{-}, v_{-}\right)$to $\left(s_{+}, v_{+}\right)$achieved by $r=0$ and any admissible control $u$ such that $\int_{\tau_{-}}^{\tau_{+}} u(\tau) d \tau=v_{+}-v_{-}$. For short, we will also make use of the notation $I$ to denote an impulse.

- $N F_{s_{0}, v_{0}}^{s_{0}^{\prime}, v_{0}}$ denotes an arc $u=0$ (no feeding) at constant volume $v_{0}$ from a concentration $s_{0}$ to a concentration $s_{0}^{\prime}<s_{0}$. For short, we will also make use of the notation $N F$ to denote an arc $u=0$.

- $S A_{i}^{v_{1}, v_{2}}$ denotes a singular $\operatorname{arc} s_{i}$ from volume $v_{1}$ to volume $v_{2}, i=1,2$.

We will say that an extremal trajectory has a switching (or switching point) at some time $t_{0}$ if it contains a sequence $I_{s_{-}, v_{-}}^{s_{+}, v_{+}} N F_{s_{+}, v_{+}}^{\bar{s}, v_{+}}$(with $s_{+}>s_{-}, \bar{s}<s_{+}$) or $N F_{s_{0}, v_{0}}^{s_{0}^{\prime}, v_{0}} I_{s_{0}^{\prime}, v_{0}}^{s_{+}, v_{+}}$(with $s_{0}^{\prime}<s_{0}, s_{+}>s_{0}^{\prime}$ ) such that the control $(r, u)$ is non-constant in a neighborhood of $t_{0}$. We now examine the possibility for an optimal control to have switching points at some point $(s, v)$ with $v<v_{m}$ and $\left.s \in\right] s_{1}, s_{2}^{*}[, i=1,2$. In the next lemma, we prove that for certain initial conditions, a trajectory reaching the singular arc $s_{2}$ with one switching point is not optimal. The proof of this lemma is illustrated on Figure 2.

Lemma 3.1. Let $\left(s_{0}, v_{0}\right) \in \mathcal{D}$ be a point such that $s_{1}^{*}<s_{0}<s_{2}$. A sequence $N F_{s_{0}, v_{0}}^{s_{-}, v_{0}} I_{s_{-}, v_{0}}^{s_{2}, v_{+}}$with $s_{-}>s_{1}^{*}$, and $v_{0}<\gamma_{2}\left(s_{-}\right)$is not optimal.

Proof. The cost of the sequence above is:

$$
J_{1}=\int_{s_{-}}^{s_{0}} \frac{d s}{\mu(s)\left(\frac{M}{v_{0}}+s_{i n}-s\right)}
$$

and we have by definition of the dilution $s_{2}=\frac{v_{0}}{v_{+}} s_{-}+\left(1-\frac{v_{0}}{v_{+}}\right) s_{i n}$. As $\mu(s) \leq \mu\left(s_{2}\right)$, we obtain

$$
J_{1} \geq \frac{1}{\mu\left(s_{2}\right)} \int_{s_{-}}^{s_{0}} \frac{d s}{\frac{M}{v_{0}}+s_{i n}-s}=\frac{1}{\mu\left(s_{2}\right)} \ln \left(\frac{M+v_{0}\left(s_{i n}-s_{-}\right)}{M+v_{0}\left(s_{i n}-s_{0}\right)}\right) .
$$


The cost of the sequence $I_{s_{0}, v_{0}}^{s_{2}, v_{2}} S A_{2}^{v_{2}, v_{+}}$is:

$$
J_{2}=\frac{1}{\mu\left(s_{2}\right)} \ln \left(\frac{M+v_{+}\left(s_{i n}-s_{2}\right)}{M+v_{2}\left(s_{i n}-s_{2}\right)}\right)
$$

where we have $s_{2}=\frac{v_{0}}{v_{2}} s_{0}+\left(1-\frac{v_{0}}{v_{2}}\right) s_{i n}$. Using $\frac{v_{0}}{v_{2}}=\frac{s_{i n}-s_{2}}{s_{i n}-s_{0}}, \frac{v_{0}}{v_{+}}=\frac{s_{i n}-s_{2}}{s_{i n}-s_{-}}$, we obtain:

$$
J_{1}-J_{2} \geq \frac{1}{\mu\left(s_{2}\right)}\left(\ln \left(\frac{M+v_{0}\left(s_{i n}-s_{-}\right)}{M+v_{0}\left(s_{i n}-s_{0}\right)}\right)-\ln \left(\frac{M+v_{+}\left(s_{i n}-s_{2}\right)}{M+v_{2}\left(s_{i n}-s_{2}\right)}\right)\right)=0,
$$

which proves the lemma.

Corollary 3.1. Any trajectory $(s(t), v(t))$ starting at some point $\left(s_{0}, v_{0}\right) \in \mathcal{D}$ with $s_{1}^{*} \leq s_{0}<s_{2}, v_{0}<v_{m}$, such that $s(t) \geq s_{1}^{*}$ for all $t$ and containing a sequence $N F_{s_{b}, v_{b}}^{s_{c}, v_{b}} I_{s_{c}}^{s_{2}, v_{b}}$, is not optimal.

Proof. It is enough to compare the trajectory with the one that coincides until the point $\left(s_{b}, v_{b}\right)$ and contains the sequence $I_{s_{b}, v_{b}}^{s_{2}, v_{+}} S A_{2}^{v_{+}, v_{c}}$. By lemma 3.1, this trajectory has a lower cost than the initial one, which concludes the proof.
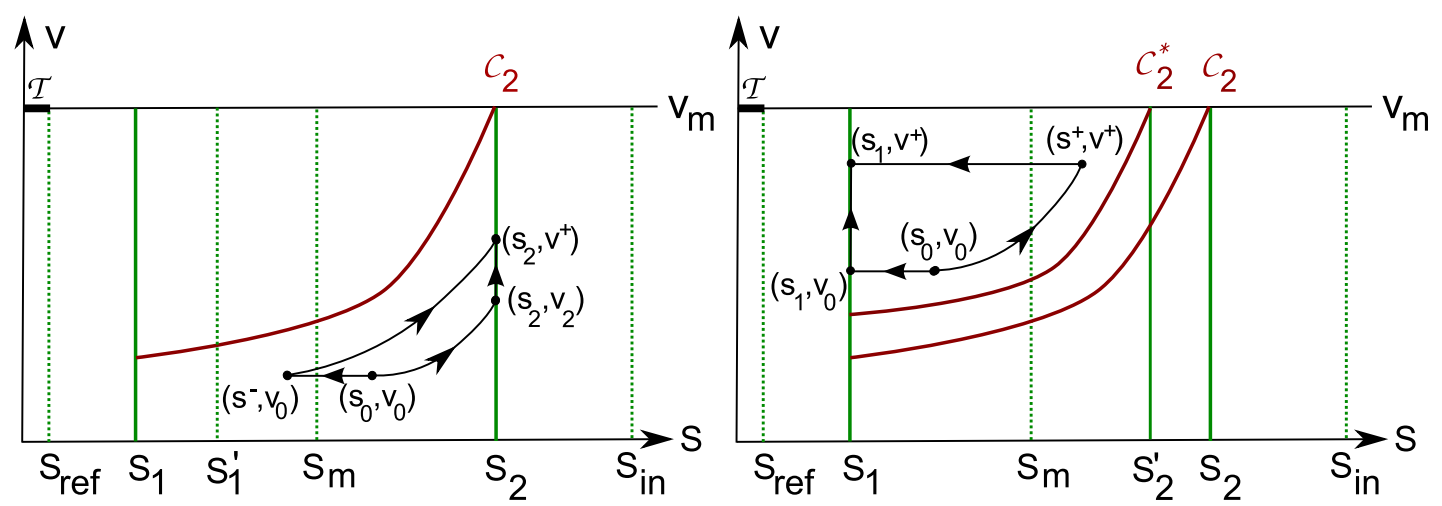

Figure 2: Figure left: illustration of the proof of lemma 3.1 for case B. Figure right: illustration of the proof of lemma 5.1 for case A.

Lemma 3.2. Let $\left(s_{0}, v_{0}\right) \in \mathcal{D}$ be a point such that $v_{0}<v_{m}, s_{0}>s_{1}$. Then, any extremal trajectory that contains a sequence $N F_{s_{0}, v_{0}}^{s_{-}, v_{0}} I_{s_{-}, v_{0}}^{s^{+}} N F_{s_{+}, v_{+}}^{s_{1}, v_{+}}$with $s_{1}<s_{-}<s_{0}$ and $s_{+} \leq s_{2}$ is not optimal.

Proof. Let $t_{s_{-}}<t_{s_{+}}$the two instants in which the sequence contains a switch. If the trajectory is optimal, we must have $\mu\left(s_{-}\right)=\mu\left(s_{+}\right)$given that $\phi_{1}\left(t_{s_{-}}\right)=\phi_{1}\left(t_{s_{+}}\right)=0$ (see lemmas 2.4 and 2.5). Consequently, one has $s_{-}<s_{m}<s_{+}$. Now, let us choose an $\varepsilon>0$ small enough such that $s_{-}-\varepsilon>s_{1}$ and $s_{+}-\varepsilon^{\prime}>s_{m}$ with $\varepsilon^{\prime}=\frac{v_{+}}{v_{0}} \varepsilon$. We will show that the cost $J_{1}^{\varepsilon}$ of the sequence $N F_{s_{0}, v_{0}}^{s_{-}, v_{0}} I_{s_{-}-\varepsilon, v_{0}}^{s^{+}-\varepsilon^{\prime}, v_{+}} N F_{s_{+}-\varepsilon^{\prime}, v_{+}}^{s_{1}, v_{+}}$is lower than the cost $J_{2}$ of the sequence $N F_{s_{0}, v_{0}}^{s_{-}, v_{0}} I_{s_{-}, v_{0}}^{s_{+}, v_{+}} N F_{s_{+}, v_{+}}^{s_{1}, v_{+}}$. Indeed, we have $\mu(s) \leq \mu\left(s_{+}\right)$for $s \in\left[s^{+}-\varepsilon^{\prime}, s_{+}\right]$and $\mu(s) \geq \mu\left(s_{-}\right)$for $s \in\left[s_{-}-\varepsilon, s_{-}\right]$so that:

$$
\begin{aligned}
J_{2}-J_{1}^{\varepsilon} & =\int_{s_{+}-\varepsilon^{\prime}}^{s_{+}} \frac{d s}{\mu(s)\left(\frac{M}{v_{+}}+s_{i n}-s\right)}-\int_{s_{-}-\varepsilon}^{s_{-}} \frac{d s}{\mu(s)\left(\frac{M}{v_{0}}+s_{i n}-s\right)} \\
& >\frac{1}{\mu\left(s_{+}\right)} \int_{s_{+}-\varepsilon^{\prime}}^{s_{+}} \frac{d s}{\frac{M}{v_{+}}+s_{i n}-s}-\frac{1}{\mu\left(s_{-}\right)} \int_{s_{-}-\varepsilon}^{s_{-}} \frac{d s}{\frac{M}{v_{0}}+s_{i n}-s} \\
& >\frac{1}{\mu\left(s_{+}\right)} \ln \left(\frac{M+v_{+}\left(s_{i n}-s_{+}+\varepsilon^{\prime}\right)}{M+v_{+}\left(s_{i n}-s_{+}\right)}\right)-\frac{1}{\mu\left(s_{-}\right)} \ln \left(\frac{M+v_{0}\left(s_{i n}-s_{-}+\varepsilon\right)}{M+v_{0}\left(s_{i n}-s_{-}\right)}\right) .
\end{aligned}
$$

Moreover, the dilution can be written:

$$
\frac{v_{0}}{v_{+}}=\frac{s_{i n}-s_{+}}{s_{i n}-s_{-}}=\frac{s_{i n}-s_{+}+\varepsilon^{\prime}}{s_{i n}-s_{-}+\varepsilon} .
$$


As $\mu\left(s_{-}\right)=\mu\left(s_{+}\right)$, we obtain $J_{2}-J_{1}^{\varepsilon}>0$, which concludes the proof.

In the following, we will focus on trajectories containing a sequence $N F S A_{1}$. Let us recall that along an arc $u=0$ starting at some point $\left(s_{0}, v_{0}\right) \in \mathcal{D}$, the switching function $\phi_{2}$ can be expressed by:

$$
\phi_{2}(t)=\phi_{2}(0)+\int_{s_{0}}^{s(t)} \psi_{v_{0}}(s) \frac{\mu^{\prime}(s)}{\mu^{2}(s)} d s
$$

and let us define a function $F$ by:

$$
(s, v) \in \mathcal{D} \longmapsto F(s, v)=\int_{s_{1}}^{s} \psi_{v}(\sigma) \frac{\mu^{\prime}(\sigma)}{\mu^{2}(\sigma)} d \sigma
$$

It follows that if $\phi_{2}<0$ until a time $t_{s_{1}}$ such that $s\left(t_{s_{1}}\right)=s_{1}$, then we have $\phi_{2}\left(t_{s_{1}}\right)=\phi_{2}(0)-F\left(s_{0}, v_{0}\right)$. We will see in the following sections that the sign of the function $F(s, v)$ is of particular interest.

Lemma 3.3. We have $F\left(s_{m}, v\right)<0$ for any volume $v$, and for given $v, s \longmapsto F(s, v)$ is increasing with respect to $s$ in the interval $\left(s_{m}, s_{2}\right)$. Moreover, for case $A$, we have:

(i). If $M>0$, then $F\left(s_{2}^{\prime}, v\right)<0$.

(ii). If $M=0$, then $F\left(s_{2}^{\prime}, v\right)=0$.

(iii). If $M<0$, then $F\left(s_{2}^{\prime}, v\right)>0$.

Proof. First, $\psi_{v}(s) \frac{\mu^{\prime}(s)}{\mu^{2}(s)}$ is negative for $s \in\left(s_{1}, s_{m}\right)$ and positive for $s \in\left(s_{m}, s_{2}\right)$, which proves that $F\left(s_{m}, v\right)<$ 0 and that $s \longmapsto F(s, v)$ is increasing with respect to $s$ in the interval $\left(s_{m}, s_{2}\right)$. Let us prove (i). Assume that the inequality does not hold. Then, we have:

$$
\int_{s_{1}}^{s_{m}} \psi_{v}(s) \frac{\mu^{\prime}(s)}{\mu^{2}(s)} d s+\int_{s_{m}}^{s_{2}^{\prime}} \psi_{v}(s) \frac{\mu^{\prime}(s)}{\mu^{2}(s)} d s \geq 0
$$

that is:

$$
I_{1}:=\int_{s_{1}}^{s_{m}} \psi_{v}(s)\left(-\frac{\mu^{\prime}(s)}{\mu^{2}(s)}\right) d s \leq \int_{s_{m}}^{s_{2}^{\prime}} \psi_{v}(s) \frac{\mu^{\prime}(s)}{\mu^{2}(s)} d s:=I_{2}
$$

Using that $\psi_{v}$ is decreasing on $\left[s_{1}, s_{2}\right]$, and $\mu\left(s_{2}^{\prime}\right)=\mu\left(s_{1}\right)$, we obtain

$$
\psi_{v}\left(s_{m}\right)\left(\frac{1}{\mu\left(s_{m}\right)}-\frac{1}{\mu\left(s_{1}\right)}\right)<I_{1} \leq I_{2}<\psi_{v}\left(s_{m}\right)\left(\frac{1}{\mu\left(s_{m}\right)}-\frac{1}{\mu\left(s_{1}\right)}\right),
$$

which is a contradiction. The proof of (iii) is analogous, given that $\psi_{v}(s)$ is increasing with $M<0$. To prove (ii), notice that $s \longmapsto \psi_{v}(s)$ is constant, so that $F\left(s_{2}^{\prime}, v\right)=0$ by $(3.2)$.

Remark 3.1. For case A, one can prove more generally that if $s \longmapsto \alpha(s)$ is a non-decreasing function (resp. $s \longmapsto \beta(s)$ is a non-increasing function), then $\int_{s_{1}}^{s_{2}^{\prime}} \alpha(s) \frac{\mu^{\prime}(s)}{\mu^{2}(s)} d s \geq 0$ (resp. $\left.\int_{s_{1}}^{s_{2}^{\prime}} \beta(s) \frac{\mu^{\prime}(s)}{\mu^{2}(s)} d s \leq 0\right)$.

\section{Optimal synthesis for case A}

In this section, we study the optimality of the singular $\operatorname{arcs} s_{1}$ and $s_{2}$ in case A.

\subsection{Preliminary results for all $M$}

In the next proposition, we prove that if a trajectory satisfies $s\left(t_{0}\right)=s_{1}$ for a certain time $t_{0}$, then it is optimal to stay on the first singular arc until the tank is completely filled.

Proposition 4.1. Let us consider an optimal trajectory such that $s(t)=s_{1}$ on some interval of time $\left[t_{0}, t_{1}\right]$. If there exists $\eta>0$ such that $s(t) \neq s_{1}$ for $\left.\left.t \in\right] t_{1}, t_{1}+\eta\right]$, then $v\left(t_{1}\right)=v_{m}$. 
Proof. Assume that $v\left(t_{1}\right)<v_{m}$. From proposition 2.3, we may discard the case $u=0$ on the interval $\left.t \in] t_{1}, t_{1}+\eta\right]$. Therefore, we can assume that $r=0$ on $\left.] t_{1}, t_{1}+\eta\right]$. In order to reach the target, there must exist a switching time $t_{2}$ with $\phi_{1}\left(t_{1}\right)=\phi_{1}\left(t_{2}\right)=0$, so $\mu\left(s_{1}\right)=\mu\left(s\left(t_{2}\right)\right.$ ) (see lemma 2.5). Because an impulse cannot cross $s_{2}$, we obtain $s\left(t_{2}\right)=s_{2}^{\prime}$. Moreover, we can prove that $s(t) \leq s_{2}^{\prime}$ by the same argument. It follows that at time $t_{2}$, we have two possibilities:

Case 1. The trajectory has a unique arc $u=0$ until $s_{1}$. In this case, the cost of the sequence $I_{s_{1}, v_{-}}^{s_{2}^{\prime}, v_{+}} N F_{s_{2}^{\prime}, v_{+}}^{s_{1}, v_{+}}$ is higher than the cost of $S A_{1}^{v_{-}, v_{+}}$. Indeed, the cost of the sequence $N F_{s_{2}^{\prime}, v_{+}}^{s_{1}, v_{+}}$is

$$
J_{1}=\int_{s_{1}}^{s_{2}^{\prime}} \frac{d s}{\mu(s)\left(\frac{M}{v_{+}}+s_{i n}-s\right)} \geq \frac{1}{\mu\left(s_{1}\right)} \ln \left(\frac{M+v_{+}\left(s_{i n}-s_{1}\right)}{M+v_{-}\left(s_{i n}-s_{2}^{\prime}\right)}\right)
$$

using $\mu(s) \leq \mu\left(s_{1}\right)$ for $s \in\left[s_{1}, s_{2}^{\prime}\right]$. Now the cost $J_{2}$ of the sequence $S A_{1}^{v_{-}, v_{+}}$is

$$
J_{2}=\frac{1}{\mu\left(s_{1}\right)} \ln \left(\frac{M+v_{+}\left(s_{i n}-s_{1}\right)}{M+v_{-}\left(s_{i n}-s_{1}\right)}\right),
$$

and using the dilution $v_{-}\left(s_{i n}-s_{1}\right)=v_{+}\left(s_{i n}-s_{2}^{\prime}\right)$, we obtain that $J_{1}-J_{2} \geq 0$.

Case 2. The trajectory has an $\operatorname{arc} u=0$, and it contains at least one more sequence $I N F$. We obtain a contradiction by lemma 3.2 (considering the last sequence $N F I N F$ before reaching $s_{1}$ ).

Finally, we prove the existence of a curve such that both strategies $S A S_{1}$ and $S A S_{2}$ are equal. Let $J_{i}(s, v)$ the cost of a singular strategy $s_{i}, i=1,2$ starting at point $(s, v)$.

Lemma 4.1. For every $\left.v_{0} \in\right] 0, v_{m}\left[\right.$, there exists at most a unique $s:=f\left(v_{0}\right)$ in $\left[s_{1}, s_{2}\right]$ such that $J_{1}\left(f\left(v_{0}\right)\right.$, $\left.v_{0}\right)=$ $J_{2}\left(f\left(v_{0}\right), v_{0}\right)$.

Proof. Let $\alpha:=\int_{s_{r e f}}^{s_{1}} \frac{d s}{\mu(s)\left(\frac{M}{v_{0}}+s_{i n}-s\right)}$. First, observe that the cost of the strategy $S A S_{2}$ is

$$
J_{2}\left(s_{0}, v_{0}\right)= \begin{cases}\int_{s_{1}}^{\frac{v_{0}}{v_{m}} s_{0}+\left(1-\frac{v_{0}}{v_{m}}\right) s_{i n}} \frac{d s}{\mu(s)\left(\frac{M}{v_{m}}+s_{i n}-s\right)}+\alpha, & \text { if } \gamma_{2}\left(s_{0}\right) \leq v_{0}, \\ \int_{s_{1}}^{s_{2}} \frac{d s}{\mu(s)\left(\frac{M}{v_{m}}+s_{i n}-s\right)}+\frac{1}{\mu\left(s_{2}\right)} \ln \left(\frac{M+v_{m}\left(s_{i n}-s_{2}\right)}{M+v_{0}\left(s_{i n}-s_{0}\right)}\right)+\alpha, & \text { if } v_{0} \leq \gamma_{2}\left(s_{0}\right),\end{cases}
$$

and that the cost of the strategy $S A S_{1}$ is:

$$
J_{1}\left(s_{0}, v_{0}\right)=\int_{s_{1}}^{s_{0}} \frac{d s}{\mu(s)\left(\frac{M}{v_{0}}+s_{i n}-s\right)}+\frac{1}{\mu\left(s_{1}\right)} \ln \left(\frac{M+v_{m}\left(s_{i n}-s_{1}\right)}{M+v_{0}\left(s_{i n}-s_{1}\right)}\right)+\alpha .
$$

For a fixed $v_{0}, s_{0} \longmapsto J_{2}\left(s_{0}, v_{0}\right)$ is continuous (both expressions of (4.1) coincide on $\mathcal{C}_{2}$ ), as is $s_{0} \longmapsto J_{1}\left(s_{0}, v_{0}\right)$; thus, $s_{0} \longmapsto g_{v_{0}}\left(s_{0}\right):=J_{2}\left(s_{0}, v_{0}\right)-J_{1}\left(s_{0}, v_{0}\right)$ is also continuous.

If $v_{0} \leq \gamma_{2}\left(s_{0}\right)$, then:

$$
g_{v_{0}}^{\prime}\left(s_{0}\right)=\frac{v_{0}}{M+v_{0}\left(s_{i n}-s_{0}\right)}\left(\frac{1}{\mu\left(s_{2}\right)}-\frac{1}{\mu\left(s_{0}\right)}\right)<0
$$

If $v_{0} \geq \gamma_{2}\left(s_{0}\right)$, then

$$
g_{v_{0}}^{\prime}\left(s_{0}\right)=\frac{v_{0}}{M+v_{0}\left(s_{i n}-s_{0}\right)}\left(\frac{1}{\mu\left(s_{+}\right)}-\frac{1}{\mu\left(s_{0}\right)}\right),
$$

where $s_{+}=\frac{v_{0}}{v_{m}} s_{0}+\left(1-\frac{v_{0}}{v_{m}}\right) s_{i n}$.

We consider three subcases:

Subcase 1: $v_{0} \leq \gamma_{2}\left(s_{1}\right)$. From equation (4.3), $s_{0} \longmapsto g_{v_{0}}\left(s_{0}\right)$ is strictly decreasing on the interval $\left[s_{1}, s_{2}\right]$.

Subcase 2: $\gamma_{2}\left(s_{1}\right)<v_{0} \leq \gamma_{2}^{*}\left(s_{1}\right)$. We obtain in this case from equation (4.4), $g_{v_{0}}^{\prime}\left(s_{0}\right)<0$ as $\mu\left(s_{+}\right)>\mu\left(s_{1}\right)>$ $\mu\left(s_{0}\right)$ for all $s_{0} \in\left[s_{1}, s_{a}\right]$, where $s_{a}=\gamma_{2}^{-1}\left(v_{0}\right)$. Moreover, $g_{v_{0}}^{\prime}\left(s_{0}\right)<0$ for all $s_{0} \in\left[s_{a}, s_{2}\right)$ (equation (4.3)). Consequently, $g_{v_{0}}\left(s_{0}\right)$ is strictly decreasing on $\left[s_{1}, s_{2}\right]$

Subcase 3: $v_{0}>\gamma_{2}^{*}\left(s_{1}\right)$. One can easily check that $g_{v_{0}}\left(s_{1}\right)>0$ (see the proof of proposition 4.1 case 1). Moreover, $g_{v_{0}}(s)$ is increasing on an interval $\left[s_{0}, \tilde{s}_{0}\right]$ where $\tilde{s}_{0} \in\left[s_{1}, \gamma_{2}^{*-1}\left(v_{0}\right)\right]$ is such that $\mu\left(\tilde{s}_{0}\right)=$ $\mu\left(\frac{v_{0}}{v_{m}} \tilde{s}_{0}+\left(1-\frac{v_{0}}{v_{m}}\right) s_{i n}\right)$, and $g_{v_{0}}(s)$ is decreasing for $s>\tilde{s}_{0}$.

Consequently, there exists at most a unique $s:=f\left(v_{0}\right)$ such that $J_{1}\left(f\left(v_{0}\right), v_{0}\right)=J_{2}\left(f\left(v_{0}\right), v_{0}\right)$. 
In the rest of the paper, we consider for $\left(s_{0}, v_{0}\right) \in \mathcal{D}$ :

$$
g_{v_{0}}\left(s_{0}\right)=J_{2}\left(s_{0}, v_{0}\right)-J_{1}\left(s_{0}, v_{0}\right),
$$

where $J_{1}\left(s_{0}, v_{0}\right)$ and $J_{2}\left(s_{0}, v_{0}\right)$ are defined by (4.2) and (4.1). We also use $\overline{\mathcal{C}}$ to denote the curve whose graph is $f$, and let $\mathcal{A}_{1}, \mathcal{A}_{2}$ be the sets defined as follows:

$$
\begin{aligned}
& \mathcal{A}_{1}=\left\{\left(s_{0}, v_{0}\right) \in \mathcal{D} \mid s_{0} \in\left[s_{1}, s_{2}\right], g_{v_{0}}\left(s_{0}\right)>0\right\}, \\
& \mathcal{A}_{2}=\left\{\left(s_{0}, v_{0}\right) \in \mathcal{D} \mid s_{0} \in\left[s_{1}, s_{2}\right], g_{v_{0}}\left(s_{0}\right)<0\right\} .
\end{aligned}
$$

For $\left(s_{0}, v_{0}\right) \in \mathcal{A}_{1}$, the strategy $S A S_{1}$ is better than $S A S_{2}$, whereas for $\left(s_{0}, v_{0}\right) \in \mathcal{A}_{1}$, the strategy $S A S_{2}$ is better than $S A S_{1}$. In Figure 3, the costs $J_{1}\left(s_{0}, v_{0}\right)$ and $J_{2}\left(s_{0}, v_{0}\right)$ have been computed using equations (4.1) and (4.2) in order to determine numerically the curve $\overline{\mathcal{C}}$.

\subsection{Case A with $M \geq 0$}

Lemma 4.2. A sequence $I_{s_{0}, v_{0}}^{s_{+}, v_{+}} N F_{s_{+}, v_{+}}^{s_{1}, v_{+}} S A_{1}^{v_{+}, v_{m}}$ such that $\left(s_{0}, v_{0}\right) \in \mathcal{D}, s_{0} \in\left(s_{1}, s_{2}\right), 0<v_{0}<v_{+}<v_{m}$, $s_{+} \in\left(s_{0}, s_{2}\right]$ is not optimal.

Proof. Let us define $J\left(v_{J}\right)$ as the cost of the sequence $I_{s_{0}, v_{0}}^{s_{J}, v_{J}} N F_{s_{J}, v_{J}}^{s_{1}, v_{J}} S A_{1}^{v_{J}, v_{m}}$, where $s_{J}=s_{J}\left(v_{J}\right)$ is defined by the dilution:

$$
\frac{v_{J}}{v_{0}}=\frac{s_{i n}-s_{0}}{s_{i n}-s_{J}\left(v_{J}\right)} .
$$

To prove this lemma, we show that $J\left(v_{J}\right)$ is minimal for $v_{J}$ such that $s_{J}\left(v_{J}\right) \in\left\{s_{0}, s_{2}\right\}$ or for $v_{J}=v_{m}$ (if $\left.v_{0}>\gamma_{2}^{*}\left(s_{0}\right)\right)$. The cost $J\left(v_{J}\right)$ can be written:

$$
J\left(v_{J}\right)=\int_{s_{1}}^{s_{J}\left(v_{J}\right)} \frac{d s}{\mu(s)\left(\frac{M}{v_{J}}+s_{i n}-s\right)}+\frac{1}{\mu\left(s_{1}\right)} \ln \left(\frac{M+v_{m}\left(s_{i n}-s_{1}\right)}{M+v_{J}\left(s_{i n}-s_{1}\right)}\right),
$$

whose derivative is:

$$
J^{\prime}\left(v_{J}\right)=\int_{s_{1}}^{s_{J}\left(v_{J}\right)} \frac{M d s}{\mu(s)\left(M+v_{J}\left(s_{i n}-s\right)\right)^{2}}+\frac{\left(s_{i n}-s_{J}\right)}{\mu\left(s_{J}\right)\left(M+v_{J}\left(s_{i n}-s_{J}\right)\right)}-\frac{\left(s_{i n}-s_{1}\right)}{\mu\left(s_{1}\right)\left(M+v_{J}\left(s_{i n}-s_{1}\right)\right)} .
$$

This can be equivalently written:

$$
J^{\prime}\left(v_{J}\right)=\int_{s_{1}}^{s_{J}\left(v_{J}\right)}-\frac{\psi_{v}^{\prime}(s)}{\mu(s)} d s+\frac{\psi_{v}\left(s_{J}\right)}{\mu\left(s_{J}\right)}-\frac{\psi_{v}\left(s_{1}\right)}{\mu\left(s_{1}\right)},
$$

and by an integration by parts, we obtain:

$$
J^{\prime}\left(v_{J}\right)=\int_{s_{1}}^{s_{J}}-\psi_{v_{J}}(s) \frac{\mu^{\prime}(s)}{\mu^{2}(s)} d s .
$$

Finally, we have:

$$
\begin{aligned}
J^{\prime \prime}\left(v_{J}\right) & =\int_{s_{1}}^{s_{J}}-\frac{\partial \psi_{v_{J}}(s)}{\partial v_{J}} \frac{\mu^{\prime}(s)}{\mu^{2}(s)} d s-\psi_{v_{J}}\left(s_{J}\right) \frac{\left(s_{i n}-s_{J}\right) \mu^{\prime}\left(s_{J}\right)}{v_{J} \mu^{2}\left(s_{J}\right)} \\
& =\int_{s_{1}}^{s_{J}} \psi_{v_{J}}^{2}(s) \frac{\mu^{\prime}(s)}{\mu^{2}(s)} d s-\psi_{v_{J}}\left(s_{J}\right) \frac{\left(s_{i n}-s_{J}\right) \mu^{\prime}\left(s_{J}\right)}{v_{J} \mu^{2}\left(s_{J}\right)} .
\end{aligned}
$$

For $M>0, s \longmapsto \psi_{v_{J}}(s)$ is decreasing, so $J^{\prime}\left(v_{J}\right)=0$ implies that:

$$
\begin{aligned}
\int_{s_{1}}^{s_{J}} \psi_{v_{J}}^{2}(s) \frac{\mu^{\prime}(s)}{\mu^{2}(s)} d s & =\int_{s_{1}}^{s_{m}} \psi_{v_{J}}^{2}(s) \frac{\mu^{\prime}(s)}{\mu^{2}(s)} d s+\int_{s_{m}}^{s_{J}} \psi_{v_{J}}^{2}(s) \frac{\mu^{\prime}(s)}{\mu^{2}(s)} d s \\
& <\psi_{v_{J}}\left(s_{m}\right) \int_{s_{1}}^{s_{m}} \psi_{v_{J}}(s) \frac{\mu^{\prime}(s)}{\mu^{2}(s)} d s+\psi_{v_{J}}\left(s_{m}\right) \int_{s_{m}}^{s_{J}} \psi_{v_{J}}(s) \frac{\mu^{\prime}(s)}{\mu^{2}(s)} d s=0
\end{aligned}
$$


consequently, $J^{\prime \prime}\left(v_{J}\right)<0$. Hence, the minimum of $J\left(v_{J}\right)$ is obtained for $v_{J}$ such that $s_{J} \in\left\{s_{0}, s_{2}\right\}$ or $v_{J}=v_{m}$ (if $\left.v_{0}>\gamma_{2}^{*}\left(s_{0}\right)\right)$.

Now for $M=0, J^{\prime}\left(v_{J}\right)=0$ is achieved if and only if $s_{J}\left(v_{J}\right)=s_{2}^{\prime}$ (by lemma 3.3). Thus, we have:

$$
J^{\prime \prime}\left(v_{J}\right)=-\psi_{v_{J}}\left(s_{2}^{\prime}\right) \frac{\left(s_{i n}-s_{2}^{\prime}\right) \mu^{\prime}\left(s_{2}^{\prime}\right)}{v_{J} \mu^{2}\left(s_{2}^{\prime}\right)}<0
$$

Thus, we can conclude as for $M>0$.

Finally, let us prove that we can exclude the case in which $v_{J}$ is such that $s_{J}=s_{2}$. If a sequence $I_{s_{0}, v_{0}}^{s_{2}, v_{J}} N F_{s_{2}, v_{J}}^{s_{1}, v_{J}}$ $S A_{1}^{v_{J}, v_{m}}$ is optimal, we have necessarily

$$
J^{\prime}\left(v_{J}\right)=\int_{s_{1}}^{s_{2}}-\psi_{v_{J}}(s) \frac{\mu^{\prime}(s)}{\mu^{2}(s)} d s=0,
$$

using that $\phi_{2}$ vanishes at the two switching points (see proof of corollary 2.1). For $M=0$, this conclusion is not possible (lemma 3.3). For $M>0$, we obtain $J^{\prime \prime}\left(v_{J}\right)<0$, so the trajectory is not optimal.

To conclude, the minimum of $J\left(v_{J}\right)$ is obtained for $v_{J}=v_{0}$ or $v_{J}=v_{m}\left(\right.$ if $\left.v_{0}>\gamma_{2}^{*}\left(s_{0}\right)\right)$. Given that $v_{0}<v_{+}<v_{m}$, the sequence $I_{s_{0}, v_{0}}^{s_{+}, v_{+}} N F_{s_{+}, v_{+}}^{s_{1}, v_{+}} S A_{1}^{v_{+}, v_{m}}$ is not optimal.

Corollary 4.1. A sequence $I_{s_{0}, v_{0}}^{s_{+}, v_{m}} N F_{s_{+}, v_{m}}^{s_{1}, v_{m}}$ such that $\left(s_{0}, v_{0}\right) \in \mathcal{D}, s_{0} \in\left(s_{1}, s_{2}^{\prime}\right), \gamma_{2}^{*}\left(s_{0}\right)<v_{0}<v_{m}$, is not optimal.

Proof. Following the same sketch as for the proof of the lemma above, we obtain:

$$
J^{\prime}\left(v_{m}\right)=\int_{s_{1}}^{s_{+}}-\psi_{v_{m}}(s) \frac{\mu^{\prime}(s)}{\mu^{2}(s)} d s=-F\left(s_{+}, v_{m}\right) .
$$

Given that $s_{+}<s_{2}^{\prime}$, we have $J^{\prime}\left(v_{m}\right)>0$ (see lemma 3.3), so the trajectory is not optimal.

Lemma 4.3. A sequence $S A_{2}^{v_{0}, v_{+}} N F_{s_{2}, v_{+}}^{s_{1}, v_{+}} S A_{1}^{v_{+}, v_{m}}$ such that $\left(s_{0}, v_{0}\right) \in \mathcal{D}, 0<v_{0}<v_{+}<v_{m}$, is not optimal.

Proof. The proof is similar to the proof of lemma 4.2. Let us define $J\left(v_{J}\right)$ as the cost of the sequence $S A_{2}^{v_{0}, v_{J}} N F_{s_{2}, v_{J}}^{s_{1}, v_{J}} S A_{1}^{v_{J}, v_{m}}$. We will show that $v_{J} \longmapsto J\left(v_{J}\right)$ reaches its minimum for $v_{J} \in\left\{v_{0}, v_{m}\right\}$. The cost $J\left(v_{J}\right)$ can be written:

$$
J\left(v_{J}\right)=\int_{s_{1}}^{s_{2}} \frac{d s}{\mu(s)\left(\frac{M}{v_{J}}+s_{i n}-s\right)}+\frac{1}{\mu\left(s_{1}\right)} \ln \left(\frac{M+v_{m}\left(s_{i n}-s_{1}\right)}{M+v_{J}\left(s_{i n}-s_{1}\right)}\right)+\frac{1}{\mu\left(s_{2}\right)} \ln \left(\frac{M+v_{J}\left(s_{i n}-s_{2}\right)}{M+v_{0}\left(s_{i n}-s_{2}\right)}\right)
$$

whose derivative is:

$$
J^{\prime}\left(v_{J}\right)=\int_{s_{1}}^{s_{2}}-\psi_{v_{J}}(s) \frac{\mu^{\prime}(s)}{\mu^{2}(s)} d s .
$$

By derivating with respect to $v_{J}$, we obtain:

$$
J^{\prime \prime}\left(v_{J}\right)=\int_{s_{1}}^{s_{2}} \psi_{v_{J}}^{2}(s) \frac{\mu^{\prime}(s)}{\mu^{2}(s)} d s .
$$

For $M>0$, if $J^{\prime}\left(v_{J}\right)=0$, then one can easily check that $J^{\prime \prime}\left(v_{J}\right)<0$. Consequently, the minimum of $J\left(v_{J}\right)$ is obtained for $v_{J} \in\left\{v_{0}, v_{m}\right\}$ (see proof of lemma 4.2).

For $M=0$, we have $J^{\prime}\left(v_{J}\right)<0$ (lemma 3.3), so the minimum is obtained for $v_{J}=v_{m}$.

Proposition 4.2. For any initial condition $\left(s_{0}, v_{0}\right) \in \mathcal{D}$ such that $s_{0} \in\left[s_{1}, s_{2}\right]$ and $0<v_{0}<v_{m}$, the optimal feedback is either $S A S_{1}$ or $S A S_{2}$.

Proof. First, from proposition 4.1, lemma 2.5 and lemma 4.3, we know that an extremal trajectory is not optimal if it leaves a singular arc $s_{i}$ before $v_{m}$. Let us now assume that there exists an optimal trajectory starting from $\left(s_{0}, v_{0}\right) \in \mathcal{D}$ and that does not coincide with one of the two singular arc strategies. This trajectory necessarily contains at least one switching point at some point $s^{*}$. We have three cases depending on whether the trajectory first reaches $s_{2}, v_{m}$, or $s_{1}$

Case 1: The trajectory reaches $s_{2}$. In this case, it contains a sequence $N F I S A_{2}$ before reaching the singular 
$\operatorname{arc} s_{2}$. We then conclude that the trajectory is not optimal by lemma 3.1 .

Case 2: The trajectory reaches $v_{m}$ (before $s_{2}$ ). In this case, it contains a sequence $N F I_{.,}^{\cdot,}, v_{m} N F_{., v_{m}}^{s_{1}, v_{m}}$ and does not reach the singular arc $s_{2}$. Using lemma 3.2, we conclude that the trajectory is not optimal.

Case 3: The trajectory reaches $s_{1}$. In this case, it contains a sequence $I N F S A_{1}$ reaching the singular arc $s_{1}$. We then conclude that the trajectory is not optimal by lemma 4.2 .
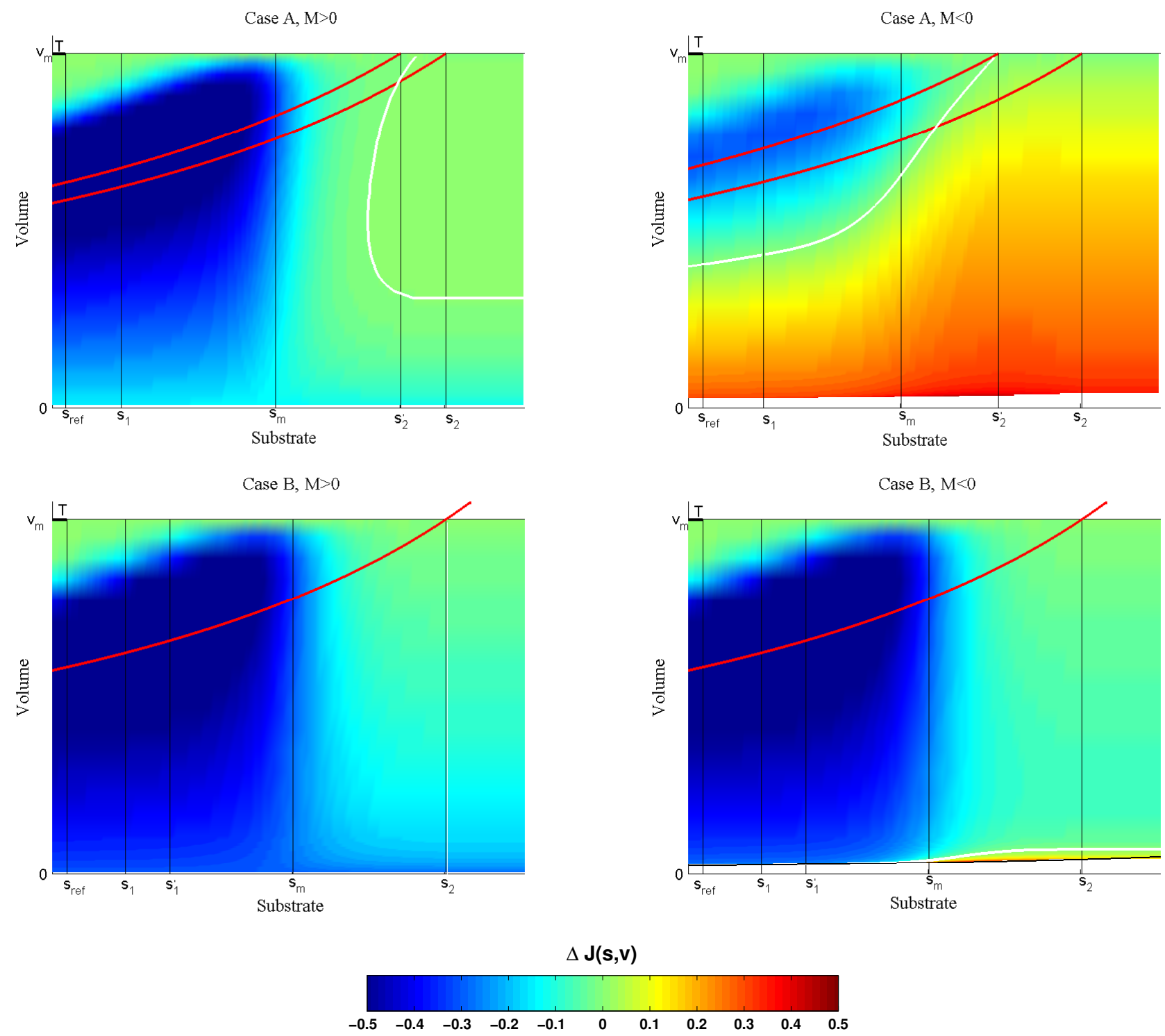

Figure 3: Comparison of the two singular arc strategies $S A S_{i}$ (of cost $J_{i}$ ). Top panels: case A, bottom panels: case B; on the left: $M>0$, on the right: $M<0$. The curve $\overline{\mathcal{C}}$ such that $J_{1}(s, v)=J_{2}(s, v)$ (see lemma 4.1) and the dilution curves in red. The background color represents $\Delta J(s, v)=\frac{J_{1}(s, v)-J_{2}(s, v)}{\max \left(J_{1}(s, v), J_{2}(s, v)\right)}$.

In this case, it remains to compare the cost of the two singular arc strategies $S A S_{1}$ and $S A S_{2}$, which are the only candidates for optimality. From lemma 4.1, the set $\mathcal{D}$ can be divided into two regions for which the optimal strategy is either $S A S_{1}$ or $S A S_{2}$. Therefore, we can conclude case A with $M \geq 0$ using the following theorem: 
Theorem 4.1. Let $\left(s_{0}, v_{0}\right) \in \mathcal{D}$.

(i). If $s_{0}<s_{1}$, then:

- If $\left(s_{1}, v_{0} \frac{s_{i n}-s_{0}}{s_{i n}-s_{1}}\right) \in \mathcal{A}_{1}$, then the optimal feedback is $S A S_{1}$.

- If $\left(s_{1}, v_{0} \frac{s_{i n}-s_{0}}{s_{i n}-s_{1}}\right) \in \mathcal{A}_{2}$, then the optimal feedback is $S A S_{2}$.

- If $f\left(v_{0} \frac{s_{i n}-s_{0}}{s_{i n}-s_{1}}\right)=s_{1}$, then the optimal feedback is either $S A S_{1}$ or $S A S_{2}$.

(ii). If $s_{0} \in\left[s_{1}, s_{2}\right]$, then:

- If $\left(s_{0}, v_{0}\right) \in \mathcal{A}_{1}$, then the optimal feedback is $S A S_{1}$.

- If $\left(s_{0}, v_{0}\right) \in \mathcal{A}_{2}$, then the optimal feedback is $S A S_{2}$.

- If $s_{0}=f\left(v_{0}\right)$, then the optimal feedback is either $S A S_{1}$ or $S A S_{2}$.

(iii). If $s_{0}>s_{2}$, then:

- If $\left(s_{2}, v_{0}\right) \in \mathcal{A}_{1}$, then the optimal feedback is $S A S_{1}$.

- If $\left(s_{2}, v_{0}\right) \in \mathcal{A}_{2}$, then the optimal feedback is $S A S_{2}$.

- If $f\left(v_{0}\right)=s_{2}$, then the optimal feedback is either $S A S_{1}$ or $S A S_{2}$.

Proof. Statement (ii) is a consequence of proposition 4.2 and lemma 4.1. Let us prove (i). From proposition 2.3, we have that $r=0$ until the concentration $s$ reaches $s=s_{1}$. Then, we can apply (ii). Similarly, for (iii), the optimal trajectory starts with $u=0$ until $s=s_{2}$ (see lemma 2.5).

Remark 4.1. Our optimal synthesis in this case shows that an extremal trajectory that has a switching point before reaching one singular arc is not optimal. In [15], the authors obtain a numerical form of the problem in this case. Extremal trajectories that contain a switching point before reaching a singular arc are depicted. We believe that the numerical approach, which is presented in [15], is very sensitive to the presence of singular arcs. Another possible explanation is that, contrary to [15], we allow impulsive control. Nevertheless, given the set of parameter values used in [15] (in particular, $u \gg \mu_{m}$ ), the impulsive problem should not differ from the numerical computations of [15] without impulse. On the other hand, in the aforementioned paper, the authors do not consider these trajectories, and they conjecture that the optimal control is either $S A S_{1}$ or $S A S_{2}$. Theorem 4.1 proves this conjecture when impulsive control is allowed.

\subsection{Case A with $M<0$}

In this section, we will consider the case A with $M<0$.

Lemma 4.4. Let $\left(s_{0}, v_{0}\right) \in \mathcal{D}$ such that $s_{2}^{\prime} \leq s_{0}<s_{\text {in }}$ and $v_{0}<v_{m}$. Then, the optimal control steering $\left(s_{0}, v_{0}\right)$ to the target $\mathcal{T}$ is $S A S_{2}$.

Proof. Let us take $s_{2}^{\prime} \leq s_{0}<s_{2}$. If we have $u=0$ at time 0 , then $\phi_{2}$ must switch at a certain time $t_{0}$ (because $\left.v_{0}<v_{m}\right)$ such that $\mu\left(s\left(t_{0}\right)\right)>\mu\left(s_{0}\right)$ (corollary 2.1). We obtain a contradiction because $\mu\left(s\left(t_{0}\right)\right) \leq \mu\left(s_{1}\right)$ and $\mu\left(s_{0}\right) \geq \mu\left(s_{2}^{\prime}\right)=\mu\left(s_{1}\right)$. Hence, we must have $r=0$ at time 0 . The same argument shows that the trajectory cannot switch to $u=0$ before reaching $s=s_{2}$. If $s_{0}>s_{2}$ and $r=0$ at the initial time, then the trajectory must switch at a certain time $t_{0}$ such that $\phi_{1}\left(t_{0}\right)=0$. We obtain $\mu\left(s\left(t_{0}\right)\right)>\mu\left(s_{0}\right)$ (lemma 2.5), which is not possible as $\mu$ is decreasing on $\left[s_{2},+\infty\right)$. Hence, we must have $u=0$ at time zero, and the same argument shows that the trajectory cannot switch before reaching $s=s_{2}$. Finally, when $s=s_{2}$, the arguments above shows that the trajectory reaches the maximum of volume $v_{m}$, which concludes the proof.

Proposition 4.3. For any volume $v \in\left(0, v_{m}\right)$, there exists a unique $s=\tilde{s}(v) \in\left(s_{m}, s_{2}^{\prime}\right)$ such that $F(\tilde{s}(v)$, $v)=$ 0 .

Proof. From lemma 3.3, the function $F(s, v)$ is continuous and increasing with respect to $s$ on $\left(s_{m}, s_{2}^{\prime}\right)$. Moreover, we have $F\left(s_{m}, v\right)<0$ and $F\left(s_{2}^{\prime}, v\right)>0$, which concludes the proof. 
In the remainder of this subsection, we will consider that the following hypothesis holds true:

Hypothesis 4.1. For any volume $v \in\left(0, v_{m}\right)$, we have:

$$
\int_{s_{1}}^{\tilde{s}(v)} \psi_{v}^{2}(s) \frac{\mu^{\prime}(s)}{\mu^{2}(s)} d s-\psi_{v}(\tilde{s}(v)) \frac{\left(s_{i n}-\tilde{s}(v)\right) \mu^{\prime}(\tilde{s}(v))}{v \mu^{2}(\tilde{s}(v))}<0 .
$$

Remark 4.2. From a geometric point of view, for a given $v$, this hypothesis holds if and only if the slope of the dilution curve passing through the point $\tilde{s}(v)$ is lower than the slope of the curve $\tilde{s}(v)$. According to various numerical simulations, this assumption seems to be always true (see figure 4).

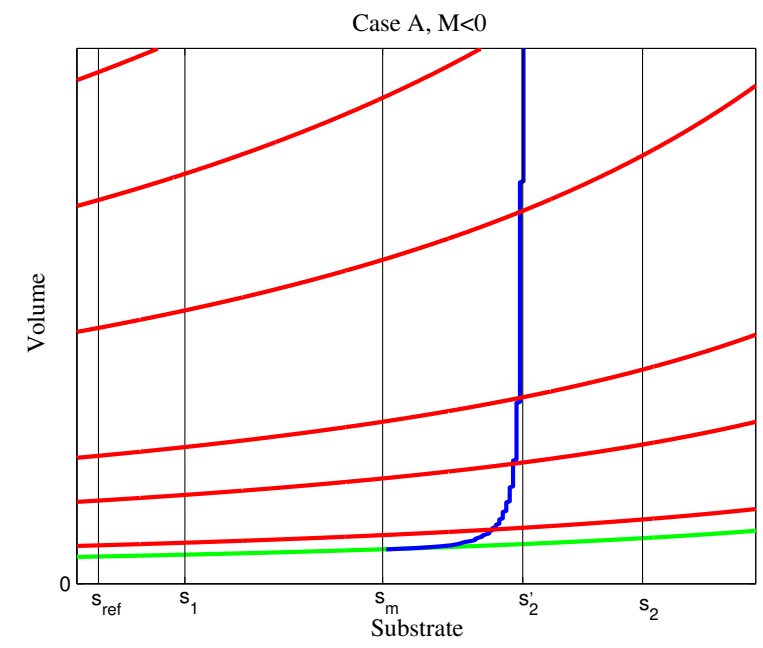

Figure 4: Verification of hypothesis 4.1. At the intersections, the slope of the dilution curves (in red) is lower than the slope of the curve $\tilde{s}(v)$ (in blue) determined numerically by solving $F(\tilde{s}(v), v)=0$ (see proposition 4.3). The green line delimits the domain $\mathcal{D}$.

Lemma 4.5. A sequence $I_{s_{0}, v_{0}}^{s_{+}, v_{+}} N F_{s_{+}, v_{+}}^{s_{1}, v_{+}} S A_{1}^{v_{+}, v_{m}}$ such that $\left(s_{0}, v_{0}\right) \in \mathcal{D}, s_{0} \in\left(s_{1}, s_{2}\right), 0<v_{0}<v_{+}<v_{m}$, $s_{+} \in\left(s_{m}, s_{2}^{\prime}\right]$ is not optimal.

Proof. As for the proof of lemma 4.2, we define $J\left(v_{J}\right)$ the cost of the sequence $I_{s_{0}, v_{0}}^{s_{J}, v_{J}} N F_{s_{J}, v_{J}}^{s_{1}, v_{J}} S A_{1}^{v_{J}, v_{m}}$. We have:

$$
J^{\prime}\left(v_{J}\right)=\int_{s_{1}}^{s_{J}}-\psi_{v_{J}}(s) \frac{\mu^{\prime}(s)}{\mu^{2}(s)} d s,
$$

and:

$$
J^{\prime \prime}\left(v_{J}\right)=\int_{s_{1}}^{s_{J}} \psi_{v_{J}}^{2}(s) \frac{\mu^{\prime}(s)}{\mu^{2}(s)} d s-\psi_{v_{J}}\left(s_{J}\right) \frac{\left(s_{i n}-s_{J}\right) \mu^{\prime}\left(s_{J}\right)}{v_{J} \mu^{2}\left(s_{J}\right)} .
$$

Given hypothesis 4.1, if $J^{\prime}\left(v_{J}\right)=0$, then $J^{\prime \prime}\left(v_{J}\right)<0$. Consequently, the minimum of $J\left(v_{J}\right)$ is obtained for $s_{J} \in\left\{s_{0}, s_{2}^{\prime}\right\}$ or $v_{J}=v_{m}$ (if $v_{0}>\gamma_{2}^{*}\left(s_{0}\right)$ ). Finally, we can exclude $s_{J}=s_{2}^{\prime}$ from lemma 4.4.

Proposition 4.4. proposition 4.2 applies for case $A$ with $M<0$.

Proof. We can follow the same proof as for proposition 4.2 with these modifications:

- It is not optimal to leave the singular arc $S A_{2}$ before $v_{m}$ from lemma 4.4.

- In case 3 , replace lemma 4.2 with lemma 4.5 
We now discuss the position of the curve $\overline{\mathcal{C}}$ in the plane $(s, v)$ (see fig. 3). Recall that $g_{v}(s)$ is defined by $g_{v}(s)=J_{2}(s, v)-J_{1}(s, v)$, where $J_{1}$ and $J_{2}$ are given by (4.1)-(4.2). From lemma 4.4, we have $g_{v_{0}}\left(s_{2}^{\prime}\right)<0$. Moreover, one can easily check that $g_{v_{0}}\left(s_{1}\right)$ is continuous and strictly increasing with respect to $v_{0}$ in the interval $\left.] 0, \gamma_{2}^{*}\left(s_{1}\right)\right]$. For $M \leq 0$, we can also show that $\lim _{v_{0} \rightarrow \frac{-M}{s_{\text {in }}-s_{1}}} g_{v_{0}}\left(s_{1}\right)=-\infty$, while $g_{\gamma_{2}^{*}\left(s_{1}\right)}\left(s_{1}\right)>0$. Consequently, there exists a unique $\left.v_{*} \in\right] \frac{-M}{s_{i n}-s_{1}}, \gamma_{2}^{*}\left(s_{1}\right)\left[\right.$ such that $J_{1}\left(s_{1}, v_{*}\right)=J_{2}\left(s_{1}, v_{*}\right)$. Hence, $\overline{\mathcal{C}}$ starts at $\left(s_{1}, v_{*}\right)$. We can now conclude case A with $M<0$.

Theorem 4.2. Let $\left(s_{0}, v_{0}\right) \in \mathcal{D}$.

(i). If $s_{0}<s_{1}$, then:

- If $v_{0} \frac{s_{i n}-s_{0}}{s_{i n}-s_{1}}>v_{*}$, then the optimal feedback is $S A S_{1}$.

- If $v_{0} \frac{s_{i n}-s_{0}}{s_{i n}-s_{1}}=v_{*}$, then the optimal feedback is either $S A S_{1}$ or $S A S_{2}$.

- If $v_{0} \frac{s_{i n}-s_{0}}{s_{i n}-s_{1}}<v_{*}$, then the optimal feedback is $S A S_{2}$.

(ii) If $s_{0} \in\left[s_{1}, s_{2}^{\prime}[\right.$, then:

- If $\left(s_{0}, v_{0}\right) \in \mathcal{A}_{1}$, then the optimal feedback is $S A S_{1}$.

- If $\left(s_{0}, v_{0}\right) \in \mathcal{A}_{2}$, then the optimal feedback is $S A S_{2}$.

- If $s_{0}=f\left(v_{0}\right)$, then the optimal feedback is either $S A S_{1}$ or $S A S_{2}$.

(iii). If $s_{0} \geq s_{2}^{\prime}$, then the optimal feedback is $S A S_{2}$.

Proof. Statement (ii) is a consequence of proposition 4.2 and lemma 4.1 and statement (iii) of lemma 4.4. Let us prove (i). From proposition 2.3, we have that $r=0$ until the concentration $s$ reaches $s=s_{1}$. Then, we can apply (ii).

\section{Optimal synthesis for case B}

In this section, we prove that $S A S_{1}$ is optimal in case B.

\subsection{Case B with $M \geq 0$}

The proof of lemma 5.1 is illustrated on Figure 2.

Lemma 5.1. A sequence $I_{s_{0}, v_{0}}^{s_{+}, v_{+}} N F_{s_{+}, v_{+}}^{s_{1}, v_{+}}$such that $\left(s_{0}, v_{0}\right) \in \mathcal{D}, s_{0} \in\left[s_{1}, s_{2}\right), 0<v_{0}<v_{m}, s_{+} \in\left(s_{0}, s_{2}\right]$ is not optimal.

Proof. We will show that the cost of the sequence $N F_{s_{0}, v_{0}}^{s_{1}, v_{0}} S A_{1}^{v_{0}, v_{+}}$, denoted $J_{2}$, is lower than the cost of the sequence above, denoted $J_{1}$ (see Figure 2). Let us write $J_{1}=J_{1}^{a}+J_{1}^{b}$ and $J_{2}=J_{2}^{a}+J_{2}^{b}$ with:

$$
J_{1}^{a}=\int_{s_{1}}^{s_{0}} \frac{d s}{\mu(s)\left(\frac{M}{v_{+}}+s_{i n}-s\right)}, J_{1}^{b}=\int_{s_{0}}^{s_{+}} \frac{d s}{\mu(s)\left(\frac{M}{v_{+}}+s_{i n}-s\right)},
$$

and

$$
J_{2}^{a}=\int_{s_{1}}^{s_{0}} \frac{d s}{\mu(s)\left(\frac{M}{v_{0}}+s_{i n}-s\right)}, J_{2}^{b}=\frac{1}{\mu\left(s_{1}\right)} \ln \left(\frac{M+v_{+}\left(s_{i n}-s_{1}\right)}{M+v_{0}\left(s_{i n}-s_{1}\right)}\right) .
$$

As $s^{+} \leq s_{2}$, we obtain:

$$
J_{1}^{b}>\frac{1}{\mu\left(s_{1}\right)} \int_{s_{0}}^{s_{+}} \frac{d s}{\frac{M}{v_{+}}+s_{i n}-s}=\frac{1}{\mu\left(s_{1}\right)} \ln \left(\frac{M+v_{+}\left(s_{i n}-s_{0}\right)}{M+v_{+}\left(s_{i n}-s_{+}\right)}\right)
$$

and, with the dilution $\frac{v_{+}}{v_{0}}=\frac{s_{i n}-s_{0}}{s_{i n}-s_{+}}$,

$$
J_{1}^{b}>\frac{1}{\mu\left(s_{1}\right)} \ln \left(\frac{M+v_{+}\left(s_{i n}-s_{0}\right)}{M+v_{0}\left(s_{i n}-s_{0}\right)}\right) .
$$


Now, let us compare $J_{1}^{a}$ and $J_{2}^{a}$. We have:

$$
J_{1}^{a}-J_{2}^{a}=\int_{s_{1}}^{s_{0}} \frac{1}{\mu(s)}\left(\frac{1}{\frac{M}{v_{+}}+s_{i n}-s}-\frac{1}{\frac{M}{v_{0}}+s_{\text {in }}-s}\right) d s .
$$

As $M \geq 0$, we have $\frac{1}{\frac{M}{v_{+}}+s_{i n}-s}-\frac{1}{\frac{M}{v_{0}}+s_{i n}-s} \geq 0$ for all $s \in\left[s_{1}, s_{0}\right]$. Therefore, we obtain:

$$
\begin{aligned}
J_{1}^{a}-J_{2}^{a} & \geq \frac{1}{\mu\left(s_{1}\right)} \int_{s_{1}}^{s_{0}}\left(\frac{1}{\frac{M}{v_{+}}+s_{i n}-s}-\frac{1}{\frac{M}{v_{0}}+s_{i n}-s}\right) d s \\
& =\frac{1}{\mu\left(s_{1}\right)} \ln \left(\frac{\left[M+v_{+}\left(s_{i n}-s_{1}\right)\right]\left[M+v_{0}\left(s_{i n}-s_{0}\right)\right]}{\left[M+v_{+}\left(s_{i n}-s_{0}\right)\right]\left[M+v_{0}\left(s_{i n}-s_{1}\right)\right]}\right) .
\end{aligned}
$$

Combining this inequality, the expression of $J_{2}^{b}$ above, and (5.1), we obtain $J_{1}-J_{2}>0$, which proves the result.

Corollary 5.1. Any trajectory $(s(t), v(t))$ starting at some point $\left(s_{0}, v_{0}\right) \in \mathcal{D}$ such that $s_{1} \leq s_{0}<s_{2}, v_{0}<v_{m}$, that is a finite concatenation of arcs $I_{s_{a}, v_{a}}^{s_{b}, v_{b}}$ and $N F_{s_{b}, v_{b}}^{s_{c}, v_{b}}$, and such that for all time $t, s_{1} \leq s(t) \leq s_{2}$, is not optimal.

Proof. As $s_{\text {ref }}<s_{1}$, there exists a time $t_{0}>0$ such that the trajectory reaches $s_{1}$ that is $s\left(t_{0}\right)=s_{1}$. Before reaching $s_{1}$, the trajectory necessarily contains a sequence: $I_{s_{a}, v_{a}}^{s_{b}, v_{b}} N F_{s_{b}, v_{b}}^{s_{1}, v_{b}}$, with $v_{a}<v_{b} \leq v_{m}$, and $s_{1} \leq s_{a}<s_{b} \leq s_{2}$. By the lemma above, the trajectory that coincides with the initial one until the point $\left(s_{a}, v_{a}\right)$ and that is then equal to $N F_{s_{a}, v_{a}}^{s_{1}, v_{1}} S A_{1}^{v_{a}, v_{b}}$, has a lower cost.

In the next corollary, we provide a region where the singular arc strategy $S A S_{2}$ is not optimal.

Corollary 5.2. Let $\left(s_{0}, v_{0}\right) \in \mathcal{D}$ such that $s_{0} \leq s_{2}$ and $0<v_{0}<v_{m}$. If $v_{0} \geq \gamma_{2}\left(s_{0}\right)$, then the singular arc strategy $\mathrm{SAS}_{2}$ is not optimal.

Proof. Given that $v_{0} \geq \gamma_{2}\left(s_{0}\right)$, the singular arc strategy $S A S_{2}$ corresponds to the sequence $I_{s_{0}, v_{0}}^{s_{+}, v_{m}} N F_{s_{+}}^{s_{1}, v_{m}}$ with $s(t)<s_{2}$ for all $t$. This corollary is therefore a direct consequence of lemma 5.1 using $v^{+}=v_{m}$.

Lemma 5.2. A sequence $S A_{2}^{v_{0}, v_{+}} N F_{s_{2}, v_{+}}^{s_{1}, v_{+}}$such that $\left(s_{0}, v_{0}\right) \in \mathcal{D}, 0<v_{0}<v_{m}$ and $v_{0}<v_{+} \leq v_{m}$ is not optimal.

Proof. Let us compare the cost of the sequence $N F_{s_{2}, v_{0}}^{s_{1}, v_{0}} S A_{1}^{v_{0}, v_{+}}$(denoted $J_{1}$ ) with the cost of the sequence $S A_{2}^{v_{0}, v_{+}} N F_{s_{2}, v_{+}}^{s_{1}, v_{+}}$(denoted $J_{2}$ ). We have $J_{1}=J_{1}^{a}+J_{1}^{b}$ and $J_{2}=J_{2}^{a}+J_{2}^{b}$ with:

$$
J_{1}^{a}=\frac{1}{\mu\left(s_{1}\right)} \ln \left(\frac{M+v_{+}\left(s_{i n}-s_{1}\right)}{M+v_{0}\left(s_{i n}-s_{1}\right)}\right), J_{1}^{b}=\int_{s_{1}}^{s_{2}} \frac{d s}{\mu(s)\left(\frac{M}{v_{0}}+s_{i n}-s\right)},
$$

and

$$
J_{2}^{a}=\frac{1}{\mu\left(s_{2}\right)} \ln \left(\frac{M+v_{+}\left(s_{i n}-s_{2}\right)}{M+v_{0}\left(s_{i n}-s_{2}\right)}\right), J_{2}^{b}=\int_{s_{1}}^{s_{2}} \frac{d s}{\mu(s)\left(\frac{M}{v_{+}}+s_{i n}-s\right)} .
$$

Let us compare $J_{1}^{b}$ and $J_{2}^{b}$ :

$$
J_{2}^{b}-J_{1}^{b}=\int_{s_{1}}^{s_{2}} \frac{1}{\mu(s)}\left(\frac{1}{\frac{M}{v_{+}}+s_{i n}-s}-\frac{1}{\frac{M}{v_{0}}+s_{i n}-s}\right) d s .
$$

Given that $M \geq 0$, we have $\frac{1}{\frac{M}{v_{+}}+s_{i n}-s}-\frac{1}{\frac{M}{v_{0}}+s_{i n}-s} \geq 0$ for all $s \in\left[s_{1}, s_{2}\right]$. Therefore, we obtain:

$$
\begin{aligned}
J_{2}^{b}-J_{1}^{b} & \geq \frac{1}{\mu\left(s_{1}\right)} \int_{s_{1}}^{s_{2}}\left(\frac{1}{\frac{M}{v_{+}}+s_{i n}-s}-\frac{1}{\frac{M}{v_{0}}+s_{i n}-s}\right) d s \\
& =\frac{1}{\mu\left(s_{1}\right)} \ln \left(\frac{\left[M+v_{+}\left(s_{i n}-s_{1}\right)\right]\left[M+v_{0}\left(s_{i n}-s_{2}\right)\right]}{\left[M+v_{+}\left(s_{i n}-s_{2}\right)\right]\left[M+v_{0}\left(s_{i n}-s_{1}\right)\right]}\right) .
\end{aligned}
$$


Using this inequality, we obtain:

$$
J_{2}-J_{1} \geq\left(\frac{1}{\mu\left(s_{2}\right)}-\frac{1}{\mu\left(s_{1}\right)}\right) \ln \left(\frac{M+v_{+}\left(s_{i n}-s_{2}\right)}{M+v_{0}\left(s_{i n}-s_{2}\right)}\right)>0,
$$

which proves the lemma.

Corollary 5.3. Let $\left(s_{0}, v_{0}\right) \in \mathcal{D}$ such that $v_{0}<\gamma_{2}\left(s_{0}\right)$. The singular arc strategy $S A S_{2}$ starting from the point $\left(s_{0}, v_{0}\right)$ is not optimal.

Proof. Because $v_{0}<\gamma_{2}\left(s_{0}\right)$, the trajectory given by the singular arc strategy $S A S_{2}$ necessarily contains a sequence $S A_{2}^{v_{1}, v_{m}} N F_{s_{2}, v_{m}}^{s_{1}, v_{m}}$, where $0<v_{1}<v_{m}$. Therefore, from the lemma above, the strategy $S A S_{2}$ is not optimal.

In the next theorem, we use the previous results to conclude that the optimal feedback is the singular arc $s_{1}$.

Theorem 5.1. Let $\left(s_{0}, v_{0}\right) \in \mathcal{D}$. For case $B$ with $M \geq 0$, the optimal strategy steering $\left(s_{0}, v_{0}\right)$ to the target is the singular arc strategy $S A S_{1}$.

Proof. First, if $s_{0}<s_{1}$, from proposition 2.3, we have that $r=0$ until the concentration $s$ reaches $s=s_{1}$. Now consider $s_{0} \geq s_{1}$. From corollary 5.2 and corollary 5.3, the singular arc strategy $S A S_{2}$ is not optimal. For the same reason, we can also eliminate all of the extremal trajectories that have at least one switching point and then reach $s_{2}$ or $v_{m}$ with a concentration $s>s_{1}$. The condition with an extremal trajectory containing a sequence of dilutions and no feeding until $s_{1}$ with $v<v_{m}$ remains to be considered. This trajectory is not optimal from lemma 5.1 and corollary 5.1. Finally, we can also exclude the case in which the trajectory leaves the singular arc $s_{1}$ with $v<v_{m}$ (consequence of lemma 2.5 and proposition 2.3(ii)). It follows that the only candidate for optimality is $S A S_{1}$.

Remark 5.1. If $M>0$, theorem 5.1 applies also for the limit case $\mu\left(s_{1}\right)=\mu\left(s_{2}\right)$. If $M=0$, lemma 5.2 no longer holds in this limit case. However, we can prove the following optimal synthesis:

- (i). If $s_{0}<s_{2}$, the optimal strategy is the singular arc strategy $S A_{1}$.

- (ii). If $s_{0} \geq s_{2}$, the optimal strategy is either $S A_{1}, S A_{2}$ or any strategy $N F_{s_{0}, v_{0}}^{s_{2}, v_{0}} S A_{2}^{v_{0}, v_{+}} N F_{s_{2}, v_{+}}^{s_{1}, v_{+}}$ $S A_{1}^{v_{+}, v_{m}} N F_{s_{1}, v_{m}}^{s_{r e f}, v_{m}}$ with $v_{+} \in\left(v_{0}, v_{m}\right)$.

Sketch of the proof. Let us prove (i). The result is clear if $s_{0}<s_{1}$ from proposition 2.3 and lemma 2.5. Now, assume that $s_{0} \in\left[s_{1}, s_{2}\right)$. The cost of a sequence $N F_{s_{+}, v_{0}}^{s_{0}, v_{0}}$ is $J_{1}=\int_{s_{0}}^{s_{+}} \frac{d s}{\mu(s)\left(s_{i n}-s\right)}$ and does not depend on $v_{0}$. Moreover, the two sequences $S A_{1}^{v_{1}, v_{2}}$ and $S A_{2}^{v_{1}, v_{2}}$ have the same cost $J_{2}=\frac{1}{\mu\left(s_{1}\right)} \ln \left(\frac{v_{2}}{v_{1}}\right)$. Finally, notice that for any $\left(s_{0}, v_{0}\right) \in \mathcal{D}$, we have $\int_{s_{0}}^{s_{+}} \frac{d s}{\mu(s)\left(s_{i n}-s\right)} \geq \frac{1}{\mu\left(s_{1}\right)} \ln \left(\frac{v_{+}}{v_{0}}\right)$, where $s_{+}=\left(1-\frac{v_{0}}{v_{+}}\right) s_{0}+\frac{v_{0}}{v_{+}} s_{i n}$. This last inequality shows that lemmas 3.1 and 5.1 hold when $M=0$. Combining these remarks yields the desired conclusion for $s_{0} \in\left[s_{1}, s_{2}\right)$.

Let us prove (ii). When $s_{0} \geq s_{2}$, the cost associated with $S A S_{1}, S A S_{2}$ and $N F_{s_{0}, v_{0}}^{s_{2}, v_{0}} S A_{2}^{v_{0}, v_{+}} N F_{s_{2}, v_{+}}^{s_{1}, v_{+}} S A_{1}^{v_{+}, v_{m}}$ $N F_{s_{1}, v_{m}}^{s_{\text {ref }}, v_{m}}$ are the same using the previous case. The conclusion follows.

\subsection{Case B with $M<0$}

For the study of this case, the following assumption will be required.

Hypothesis 5.1. For a given $v_{0}$ such that $0<v_{0}<v_{m}$, we have:

$$
\left(\frac{1}{\mu\left(s_{m}\right)}-\frac{1}{\mu\left(s_{1}\right)}\right) \psi_{v}\left(s_{1}\right)>\left(\frac{1}{\mu\left(s_{m}\right)}-\frac{1}{\mu\left(s_{2}\right)}\right) \psi_{v}\left(s_{2}\right),
$$

for all $v \in\left[v_{0}, v_{m}\right)$.

Remark 5.2. One can easily check that this hypothesis holds when $M \rightarrow 0^{-}\left(\right.$as $\left.\psi_{v}(s) \rightarrow \frac{1}{v}\right)$.

Lemma 5.3. Under hypothesis 5.1, lemma 5.2 and corollary 5.3 hold for $M<0$. 
Proof. We show that one can find a volume $v_{-} \in\left[v_{0}, v_{+}\left[\right.\right.$such that the cost of the trajectory $N F_{s_{2}}^{s_{1}, v_{-}} S A_{1}^{v_{-}, v_{+}}$ is lower than the cost of the sequence $S A_{2}^{v_{-}, v_{+}} N F_{s_{2}, v_{+}}^{s_{1}, v_{+}}$. Consider $\varepsilon=v_{+}-v_{-}>0$. Notice that $v_{+}$is fixed, and $v_{-}=v_{-}(\varepsilon)$ is a function of $\varepsilon$; therefore, we denote by $J_{1}(\varepsilon)$ the cost of $N F_{s_{2}, v_{-}}^{s_{1}, v_{-}} S A_{1}^{v_{-}, v_{+}}$and $J_{2}(\varepsilon)$ the cost of $S A_{2}^{v_{-}, v_{+}} N F_{s_{2}, v_{+}}^{s_{1}, v_{+}}$. Let us write $J_{1}(\varepsilon)=J_{1}^{a}(\varepsilon)+J_{1}^{b}(\varepsilon)$ and $J_{2}(\varepsilon)=J_{2}^{a}(\varepsilon)+J_{2}^{b}(\varepsilon)$ with:

$$
J_{1}^{a}(\varepsilon)=\frac{1}{\mu\left(s_{1}\right)} \ln \left(\frac{M+v_{+}\left(s_{i n}-s_{1}\right)}{M+v_{-}\left(s_{i n}-s_{1}\right)}\right), J_{1}^{b}(\varepsilon)=\int_{s_{1}}^{s_{2}} \frac{d s}{\mu(s)\left(\frac{M}{v_{-}}+s_{i n}-s\right)},
$$

and

$$
J_{2}^{a}(\varepsilon)=\frac{1}{\mu\left(s_{2}\right)} \ln \left(\frac{M+v_{+}\left(s_{i n}-s_{2}\right)}{M+v_{-}\left(s_{i n}-s_{2}\right)}\right), J_{2}^{b}(\varepsilon)=\int_{s_{1}}^{s_{2}} \frac{d s}{\mu(s)\left(\frac{M}{v_{+}}+s_{i n}-s\right)} .
$$

Given that for $i=1,2$ :

$$
\frac{M+v_{+}\left(s_{i n}-s_{i}\right)}{M+v_{-}\left(s_{i n}-s_{i}\right)}=1+\varepsilon \psi_{v_{-}}\left(s_{i}\right)
$$

we obtain $J_{2}^{a}(\varepsilon)-J_{1}^{a}(\varepsilon) \sim a \varepsilon$ when $\varepsilon$ goes to zero, where

$$
a=\frac{\psi_{v_{+}}\left(s_{2}\right)}{\mu\left(s_{2}\right)}-\frac{\psi_{v_{+}}\left(s_{1}\right)}{\mu\left(s_{1}\right)} .
$$

Clearly $J_{2}^{b}(\varepsilon)-J_{1}^{b}(\varepsilon) \rightarrow 0$ when $\varepsilon$ goes to zero. By a Taylor expansion, we obtain when $\varepsilon$ goes to zero: $J_{2}^{b}(\varepsilon)-J_{1}^{b}(\varepsilon) \sim b \varepsilon$, with:

$$
b:=\int_{s_{1}}^{s_{2}} \frac{M}{\mu(s)\left(M+v_{+}\left(s_{i n}-s\right)\right)^{2}} d s=\int_{s_{1}}^{s_{2}}-\frac{\psi_{v_{+}}^{\prime}(s)}{\mu(s)} d s .
$$

As $M<0$, we obtain the inequality:

$$
b>\frac{1}{\mu\left(s_{m}\right)}\left(\psi_{v_{+}}\left(s_{1}\right)-\psi_{v_{+}}\left(s_{2}\right)\right)
$$

Combining these expansions yields

$$
\liminf _{\varepsilon \rightarrow 0^{+}}\left(J_{2}(\varepsilon)-J_{1}(\varepsilon)\right) \geq \liminf _{\varepsilon \rightarrow 0^{+}}(a+b) \varepsilon \geq 0,
$$

the last inequality above is a consequence of hypothesis 5.1 with $v=v_{+}$. This concludes the proof for lemma 5.2. The corollary is a direct consequence of the lemma.

Proposition 5.1. For any volume $v \in\left(0, v_{m}\right)$, there exists at most a unique $s=\tilde{s}(v) \in\left(s_{m}, s_{2}^{\prime}\right)$ such that $F(\tilde{s}(v), v)=0$.

Proof. For fixed $v$, the function $s \longmapsto F(s, v)$ is continuous and increasing with respect to $s$ on $\left(s_{m}, s_{2}^{\prime}\right)$. Moreover, we have $F\left(s_{m}, v\right)<0$, which concludes the proof.

As for case A with $M<0$, we have to introduce the following hypothesis:

Hypothesis 5.2. For any volume $v \in\left(0, v_{m}\right)$, if $\tilde{s}(v)$ exists, then we have:

$$
\int_{s_{1}}^{\tilde{s}(v)} \psi_{v}^{2}(s) \frac{\mu^{\prime}(s)}{\mu^{2}(s)} d s-\psi_{v}(\tilde{s}(v)) \frac{\left(s_{i n}-\tilde{s}(v)\right) \mu^{\prime}(\tilde{s}(v))}{v \mu^{2}(\tilde{s}(v))}<0 .
$$

Lemma 5.4. Under hypothesis 5.2, a sequence $I_{s_{0}, v_{0}}^{s_{1}, v_{+}} N F_{s_{+}, v_{+}}^{s_{1}, v_{+}} S A_{1}^{v_{+}, v_{m}}$ such that $\left(s_{0}, v_{0}\right) \in \mathcal{D}, s_{0} \in\left(s_{1}, s_{2}\right)$, $0<v_{0}<v_{+}<v_{m}, s_{+} \in\left(s_{m}, s_{2}\right]$ is not optimal.

Proof. The proof is the same as the proof of lemma 4.5 .

Lemma 5.5. The singular arc strategy $S A S_{2}$ is not optimal.

Proof. Recall that 
- if $v_{0} \geq \gamma_{2}\left(s_{0}\right)$, then $g_{v_{0}}^{\prime}\left(s_{0}\right)=\frac{v_{0}}{M+v_{0}\left(s_{i n}-s_{0}\right)}\left(\frac{1}{\mu\left(s_{+}\right)}-\frac{1}{\mu\left(s_{0}\right)}\right)$, where $s_{+}=\frac{v_{0}}{v_{m}} s_{0}+\left(1-\frac{v_{0}}{v_{m}}\right) s_{i n}$

- if $v_{0} \leq \gamma_{2}\left(s_{0}\right)$, then $g_{v_{0}}^{\prime}\left(s_{0}\right)=\frac{v_{0}}{M+v_{0}\left(s_{i n}-s_{0}\right)}\left(\frac{1}{\mu\left(s_{2}\right)}-\frac{1}{\mu\left(s_{0}\right)}\right)$.

Hence, for a given volume $v_{0}>\gamma_{2}\left(s_{1}^{\prime}\right), s \longmapsto g_{v_{0}}(s)$ is a continuous function that is increasing on the interval $\left[s_{1}^{\prime}, \tilde{s}_{0}\right]$ where $\tilde{s}_{0} \in\left[s_{1}^{\prime}, s_{2}\right]$ is such that $\mu\left(\tilde{s}_{0}\right)=\mu\left(\frac{v_{0}}{v_{m}} \tilde{s}_{0}+\left(1-\frac{v_{0}}{v_{m}}\right) s_{i n}\right)$; and is decreasing for $s>\tilde{s}_{0}$.

Now, let us assume $s_{0}<s_{1}^{\prime}$. The result is then a direct consequence of lemma 2.5. Assume now that $s_{0} \geq s_{1}^{\prime}$. If $v_{0}<\gamma_{2}\left(s_{0}\right), S A_{2}$ is not optimal from lemma 5.3. The case for which $v_{0}>\gamma_{2}\left(s_{0}\right)$ remains to be considered. One has $g_{v_{0}}\left(s_{1}^{\prime}\right)>0$ as the singular arc strategy and that $S A S_{1}$ is optimal in this case (lemma 2.5), and we also have $g_{v_{0}}\left(s_{2}\right)>0$ from lemma 5.3. Consequently, given that $g_{v_{0}}(s)$ is increasing on $\left[s_{1}^{\prime}, \tilde{s}_{0}\right]$ and decreasing on $\left[\tilde{s}_{0}, s_{2}\right]$, we have $g_{v_{0}}(s)>0, \forall s \in\left[s_{1}^{\prime}, s_{2}\right]$, and the singular arc strategy $S A S_{2}$ is not optimal.

Finally, we can conclude the case B with $M<0$ as follows.

Theorem 5.2. Let $\left(s_{0}, v_{0}\right) \in \mathcal{D}$. For case $B$ with $M<0$, under hypotheses 5.1 and 5.2, the optimal strategy steering $\left(s_{0}, v_{0}\right)$ to the target is the singular arc strategy $S A S_{1}$.

Proof. The proof is similar to the proof of theorem 5.1, eliminating the singular arc strategy $S A S_{2}$ (lemma 5.5 ) and all of the trajectories containing a sequence of dilution and no feeding until $s_{1}$ with $v<v_{m}$. (lemma $5.4)$.

Remark 5.3. In contrast to what was stated in the previous theorem, we can see in Figure 3 a region where $S A S_{2}$ is better than $S A S_{1}$. Indeed, hypothesis 5.2 does not hold in this region. Moreover, this area has no practical interest because it corresponds to very low levels of $x$.

\section{Conclusion}

In this work, the problem of the minimal time control of a fed-batch bioreactor has been considered for a growth function with two local maxima defining two competitive singular arc strategies. Using a numerical approach, Rapaport and Dochain [15] have conjectured that the state space can be divided in two domains in which each arc strategy is optimal. In the present work, we provide the optimal synthesis for this problem allowing for impulsive controls to provide an answer to the question stated in [15]. These results are obtained using the Pontryagin maximum principle to derive the necessary conditions on optimal trajectories. In particular, our method relies on the analysis of the switching functions and on the cost comparison of extremal trajectories in order to exclude certain strategies.

\section{Acknowledgements}

This work was supported by the Programa de Financiamiento Basal from the Center of Mathematical Modeling, Universidad de Chile and was developed in the context of DYMECOS INRIA associated team and of the program Stic-AmSud MOMARE. The first author thanks the team DYMECOS for partial financial support. P. Gajardo and F. Mairet were partially supported by the FONDECYT-Chile program (N 1080173 and N 3120117, respectively).

\section{References}

[1] J. F. AndREWs, A mathematical model for the continuous culture of microorganisms utilizing inhibitory substrates, Biotechnology Bioengineering, vol. 10, pp. 707-723, 1968.

[2] T. Bayen, P. Gajardo, F. Mairet, Minimal time control of fed-batch bioreactor for growth functions with product inhibition, under preparation.

[3] M.J. Betancur, J.A. Moreno, I. Moreno-Andrade, G. Buitrón, Practical optimal control of fedbatch bioreactors for the waste water treatment, International Journal of Robust and Nonlinear Control, vol. 16, 3, pp. 173-190, 2006. 
[4] Cesari, Lamberto, Optimization-Theory and Applications. Problems with ordinary differential equations, Springer, 1983.

[5] P. Cougnon, D. Dochain, M. Guay, M. Perrier, On-line optimization of fedbatch bioreactors by adaptive extremum seeking control, Journal of Process Control, vol. 21, 10, pp. 1526-1532, 2011.

[6] P.M. Doran, Bioprocess engineering principles, Academic Press, 1995.

[7] P. Gajardo, H. Ramirez, A. Rapaport, Minimal time sequential batch reactors with bounded and impulse controls for one or more species, SIAM J. Control Optim., Vol. 47, 6, pp. 2827-2856, 2008.

[8] J. Hong, Optimal substrate feeding policy for a fed batch fermentation with substrate and product inhibition kinetics, Biotechnology and bioengineering, 28, pp. 1421-1431, 1986.

[9] J. Lee, S.Y. Lee, S. Park, A.P.J. Middelberg, Control of fed-batch fermentations, Biotechnology advances, Vol. 17, 1, pp. 29-48, 1999.

[10] C. Liu, Optimal control for nonlinear dynamical system of microbial fed-batch culture, Journal of computational and applied mathematics, 232, pp. 252-261, 2009.

[11] A. Miele, Application of Green's Theorem to the extremization of linear integrals, Symp. on Vehicle Systems Optimization, Garden City, L. I. New York, pp. 26-35, 1961.

[12] J. Monod, Recherches sur la Croissance des Cultures Bactériennes, Hermann, Paris 1942.

[13] J. A. Moreno, Optimal time control of bioreactors for the wastewater treatment, Optim. Control Appl. Meth., 20, pp. 145-164, 1999.

[14] L.S. Pontryagin, V.G. Boltyanskiy, R.V. Gamkrelidze, E.F. Mishchenko, Mathematical theory of optimal processes, The Macmillan Company, 1964.

[15] A. Rapaport, D. Dochain, , Minimal time control of fed-batch processes for growth functions with several maxima, IEEE Transactions on Automatic Control, vol. 56, 11, pp. 2671-2676, 2011.

[16] H. M. Robbins, A Generalized Legendre-Clebsch Condition for the Singular Cases of Optimal Control, IBM J. of Research and Development 11, pp. 361-372, 1967.

[17] H. L. Smith, P. Waltman, The theory of the chemostat, Dynamics of microbial competition, cambridge University Press, 1995. 